\title{
Development and characterization of microsatellite markers for Morus spp. and assessment of their transferability to other closely related species
}

Balachandran Mathithumilan ${ }^{1 \dagger}$, Niteen Narharirao Kadam ${ }^{1 \dagger}$, Jyoti Biradar ${ }^{2}$, Sowmya H Reddy ${ }^{1}$, Mahadeva Ankaiah ${ }^{1}$, Madhura J Narayanan', Udayakumar Makarla', Paramjit Khurana ${ }^{3}$ and Sheshshayee Madavalam Sreeman ${ }^{{ }^{*}}$

\begin{abstract}
Background: Adoption of genomics based breeding has emerged as a promising approach for achieving comprehensive crop improvement. Such an approach is more relevant in the case of perennial species like mulberry. However, unavailability of genomic resources of co-dominant marker systems has been the major constraint for adopting molecular breeding to achieve genetic enhancement of Mulberry. The goal of this study was to develop and characterize a large number of locus specific genic and genomic SSR markers which can be effectively used for molecular characterization of mulberry species/genotypes.

Result: We analyzed a total of 3485 DNA sequences including genomic and expressed sequences (ESTs) of mulberry (Morus alba L.) genome. We identified 358 sequences to develop appropriate microsatellite primer pairs representing 222 genomic and 136 EST regions. Primers amplifying locus specific regions of Dudia white (a genotype of Morus alba L), were identified and 137 genomic and 51 genic SSR markers were standardized. A two pronged strategy was adopted to assess the applicability of these SSR markers using mulberry species and genotypes along with a few closely related species belonging to the family Moraceae viz., Ficus, Fig and Jackfruit. While $100 \%$ of these markers amplified specific loci on the mulberry genome, $79 \%$ were transferable to other related species indicating the robustness of these markers and the potential they hold in analyzing the molecular and genetic diversity among mulberry germplasm as well as other related species. The inherent ability of these markers in detecting heterozygosity combined with a high average polymorphic information content (PIC) of 0.559 ranging between 0.076 and 0.943 clearly demonstrates their potential as genomic resources in diversity analysis. The dissimilarity coefficient determined based on Neighbor joining method, revealed that the markers were successful in segregating the mulberry species, genotypes and other related species into distinct clusters.

Conclusion: We report a total of 188 genomic and genic SSR markers in Morus alba L. A large proportion of these markers (164) were polymorphic both among mulberry species and genotypes. A substantial number of these markers (149) were also transferable to other related species like Ficus, Fig and Jackfruit. The extent of polymorphism revealed and the ability to detect heterozygosity among the cross pollinated mulberry species and genotypes render these markers an invaluable genomic resource that can be utilized in assessing molecular diversity as well as in QTL mapping and subsequently mulberry crop improvement through MAS.
\end{abstract}

\footnotetext{
* Correspondence: msheshshayee@hotmail.com

${ }^{\dagger}$ Equal contributors

'Department of Crop Physiology, University of Agricultural Sciences,

Bangalore, India

Full list of author information is available at the end of the article
} 


\section{Background}

Mulberry, a perennial out-breeding tree species is distributed in varied environments ranging from tropical to sub-arctic regions. The wide distribution can be attributed to its capability to adapt to diverse agro-climatic conditions, fast regeneration and both sexual and asexual modes of propagation. The mulberry leaf serves as the sole source of food to the domesticated silkworm, Bombyxmori L., and hence contributes significantly to the success of silk industry in India. It is predicted that around 27,000 MT of raw silk would need to be produced by the year 2030 to meet the demand in India [1]. This goal is strongly dependant on improving mulberry productivity. Enhancing the yield potential and minimizing the yield loss due to stresses are therefore the most viable strategies to achieve genetic enhancement of mulberry [2].

Despite the significant progress achieved so far, genetic improvement of mulberry yield potential through conventional breeding has been distressingly slow, mainly because of the perennial growth habit and complex inheritance pattern. Convincing evidences suggest that relevant traits need to be introgressed onto an elite genetic background to achieve greater success in crop improvement endeavors. Thus, the applications of modern molecular and genomic tools are expected to strongly complement the breeding efforts in enhancing yield potential of mulberry [2]. Advances in PCR based genomic approaches have generated robust DNA marker systems $[3,4]$, which offer an effective approach to augment breeding methods for mulberry improvement [5]. Randomly amplified polymorphic DNA (RAPD), Amplified fragment length polymorphism (AFLP) and Inter simple sequence repeats (ISSR) have been the most frequently employed marker systems to study the genetic diversity among mulberry species and genotypes [6-8]. Though these marker systems provide a good option to discriminate the evolutionary relationships among species [9], being dominant, RAPD, AFLP and ISSR markers have limited application in marker assisted breeding, especially in heterozygous out-breeding perennial species like mulberry. Lack of sufficient number of co-dominant marker systems renders molecular breeding practices in mulberry still a distant possibility.

Microsatellites or simple sequence repeats (SSR) are short stretches of tandemly repeated DNA sequences, distributed throughout the eukaryotic genome $[10,11]$. SSR markers display locus specificity, are co-dominant and highly transferable to other related species [12] and hence are the most attractive choice of marker systems for mulberry. Further, the higher ability to detect polymorphism by the SSR markers is an added advantage while analyzing closely related species and/or genotypes, which is often the case in breeding programs [13]. The efficiency of the SSR markers in genetic screening has been reported in tree species like peach, olive and fig [14-16].

Except for the reports of Aggarwal et al. [17] and Zhao et al. [18], there have not been many efforts in developing co-dominant markers in mulberry. From this background, the main aim of this work was to generate SSR markers for characterizing mulberry germplasm and/or mapping populations. We report a large number of genic and genomic SSR markers for mulberry and examined their transferability to closely related species like Ficus (Ficusbengalensis), Fig (Ficuscarica) and Jackfruit (Artocarpusheterophyllus).

\section{Result and discussion}

Pre-cloning enrichment strategy was adopted to isolate the genomic microsatellite regions and a set of previously characterized expressed sequence tags (ESTs) [19-21] were analyzed to identify genic microsatellite regions. A total of 3485 sequences, including 1094 genomic and 2391 EST sequences were analyzed for the presence of microsatellite regions. Locus specific primers were designed for such target sequences to develop SSR markers.

\section{Isolation and characterization of genomic microsatellites}

Analysis of the genomic sequences revealed a total of 900 diverse microsatellite loci (Table 1). Among them, 167 (18.56\%) sequences had mono nucleotide repeats (MNR) followed by 303 (33.67\%) sequences with dinucleotide repeats (DNR). Tri nucleotide repeats (TNR) were found among $155(17.22 \%)$ sequences while tetra (TtNR), penta (PNR) and hexa (HNR) nucleotide repeats were relatively less frequent in the enrichment library (Figure 1). Besides these types, 52 (5.78\%) microsatellite loci with repeat motifs having more than six nucleotide bases referred to as long nucleotide repeats (LNR) were also identified. It is well accepted that di, tri, tetra, penta and hexa repeat motifs represent an appropriate marker system and can generally distinguish greater diversity [22]. Hence, the LNRs and MNRs were excluded from designing locus specific primers. In our study, "TC/AG" repeats constituted the most frequent DNR microsatellite variant (25.5\%) followed by "CT/GA". While "AT/TA" and "AG/TC" repeats were reported as the most frequent in plant genomes [17,23-29]. He et al. [30] identified "GA/CT" as the most frequently occurring di-repeat motifs in groundnut. Our results revealed the presence of both the types of DNR motifs indicating a possibility that these markers would be able to distinguish greater diversity among mulberry accessions. The least abundant DNR motifs found in genomic SSRs was "CA/GT and $\mathrm{CG} / \mathrm{GC}$ ". The frequency of "GC" repeats was generally less in genomic regions of most plants as reported in peach [31], coffee [32], rubber tree [33], wheat [34] and soybean [35]. While "GAA" repeats were most 
Table 1 Sequences analyzed while developing genomic and genic SSR markers in mulberry

\begin{tabular}{llllllll}
\hline Library & $\begin{array}{l}\text { No. of colonies } \\
\text { screened }\end{array}$ & $\begin{array}{l}\text { No. of clones } \\
\text { sequenced/Transcripts } \\
\text { screened }\end{array}$ & $\begin{array}{l}\text { Clones with } \\
\text { SSR repeats }\end{array}$ & $\begin{array}{l}\text { Sequences containing } \\
\text { more than one SSR }\end{array}$ & $\begin{array}{l}\text { Total no. } \\
\text { of repeats }\end{array}$ & $\begin{array}{l}\text { Primers } \\
\text { developed }\end{array}$ & $\begin{array}{l}\text { Primers standardized/Locus } \\
\text { specific amplification }\end{array}$ \\
\hline Genomic & 1588 & 1094 & 484 & 234 & 900 & 222 & 137 \\
EST & - & 2391 & 800 & 254 & 1155 & 136 & 51 \\
Total & 3485 & 1284 & 488 & 2055 & 358 & 188 \\
\hline
\end{tabular}

frequent (15.9\%) among the TNRs, "AAAT" repeats were the most frequent tetra nucleotide repeats (16.6\%). Similarly, "AAAAC" and "AAAAAG" repeat types were more frequent among the PNR and HNR groups, respectively.

Based on the repeat sequences, the microsatellite regions were classified as perfect, interrupted (more than one of the same repeat motif spaced by a few base pairs) and compound repeats (different repeat motifs occurring tandemly and/or interrupted by a few base pairs). Details about the genomic SSR marker types, their repeat motifs detected in the enrichment library and the gene bank accession number are presented in Table 2 . Of the repeat regions identified, $74.5 \%$ were perfect, $6.5 \%$ were interrupted and 19\% were compound repeats. Repeat regions of the "perfect" type are more common in plant genome compared with "interrupted" or "compound" [36,37]. Though greater representation of compound repeat motifs is not common in plant genomes, they seem to exhibit greater levels of polymorphism and hence have a distinct advantage in mapping and diversity analysis [38-41].

\section{Development of genomic SSR markers}

Although DNA sequences harboring microsatellite regions were captured using specific probes, primers could not be designed to all the sequences. In instances where the repeat stretch was less than 15 nucleotides or in situations where the repeat regions were close to the ends of the sequences, primers were not designed. Thus, out of the 1094 genomic clones sequenced, 222 primer pairs could be developed (Table 1). The web-based program, Primer3 (http://bioinfo.ebc.ee/mprimer3/), was adopted to design primers to the identified regions with more than 15 nucleotide repeats so as to amplify at least $150 \mathrm{bp}$ fragments. The pre-cloning enrichment strategy captured specific genomic regions that were complementary to the microsatellite probes used. Thus, this approach enhanced the success of identifying specific loci that were unique in the genome. Of the set of 222 primer pairs developed, 137 (61.71\%) showed locus specific amplification reiterating the advantages of the pre-cloning enrichment strategy in discovering microsatellite regions [17,30,42,43]. These locus specific markers

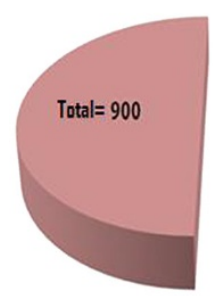

GenomicSSR

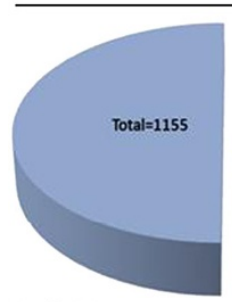

Genic SSR

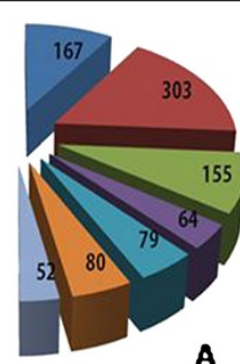

A

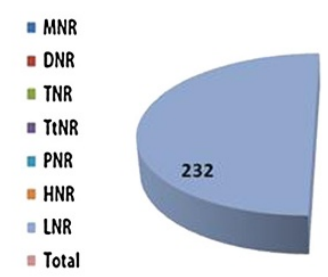

- Total

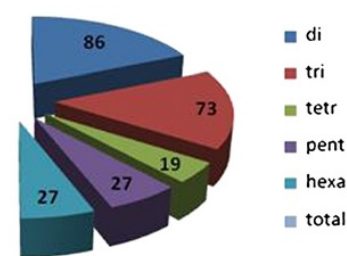

A1 tri

tetr

hexa

total

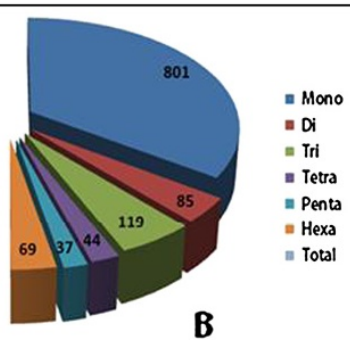

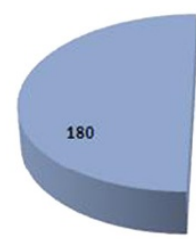

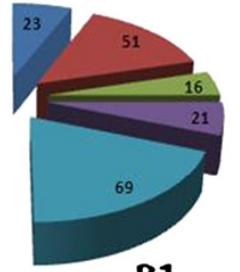

B1 a di

- tri

= tetr

a pent

a hexa

= total

Figure 1 Classification and diversity of repeat types among the identified genomic and genic microsatellite motifs. The total number of microsatellite motifs on genomic sequences is illustrated in panel $\mathbf{A}$ while the genic microsatellites are in $\mathbf{B}$. The locus specific marker diversity of genomic and genic microsatellites is illustrated in $\mathbf{A} \mathbf{1}$ and $\mathbf{B} \mathbf{1}$, respectively. 
Table 2 Details of the genomic SSR markers developed for mulberry

\begin{tabular}{|c|c|c|c|c|c|c|c|}
\hline SI no & Primer name & Primer sequence & GenBank-ID & Amplicon size & Repeat motif & Ta $\left({ }^{\circ} \mathrm{C}\right)$ & Repeat type \\
\hline \multirow[t]{2}{*}{1} & MulSSRIF & GATCTGAAGTCACCCAGCC & GF101960 & 236 & TC & 56.8 & Perfect \\
\hline & MulSSRIR & GCAGAATCTITTCAGCTTCCA & & & & & \\
\hline \multirow[t]{2}{*}{2} & MulSSR2F & GGTGCCTGAAGATATGTGG & BV722881 & 154 & $A C$ & 56.8 & Perfect \\
\hline & MulSSR2R & CTCTGAGGGAAGCAGAAG & & & & & \\
\hline \multirow[t]{2}{*}{3} & MulSSR23F & CGGAAACAGCCCAAAGAAGG & GF101977 & 223 & AAACCT & 56.8 & Perfect \\
\hline & MulSSR23R & AGGAGGGGTTTAGGGG & & & & & \\
\hline \multirow[t]{2}{*}{4} & MulSSR26F & CCACTGGTGCCTGAAG & BV722891 & 282 & $A C$ & 56.8 & Perfect \\
\hline & MulSSR26R & CATCTCATACTGGGGC & & & & & \\
\hline \multirow[t]{2}{*}{5} & MulSSR-82 F & CAATCACTAACGGGGGAAG & BV722895 & 240 & CT & 56.8 & Perfect \\
\hline & MulSSR-82R & GCTCTITITGGTGCTCC & & & & & \\
\hline \multirow[t]{2}{*}{6} & MULSSR59F & GGTTCATTTCCCTCTCGA & BV722893 & 243 & TTC & 56.8 & Perfect \\
\hline & MULSSR59R & GGCCGATGCGAACAGA & & & & & \\
\hline \multirow[t]{2}{*}{7} & MULSSR85F & CCGGAGAAATTCCAAAGG & BV722896 & 304 & TC & 56.8 & Perfect \\
\hline & MULSSR85R & CATCCAGGCATCTGATTG & & & & & \\
\hline \multirow[t]{2}{*}{8} & MULSSR69F & CAATATTACCACCCTCAC & GF101963 & 294 & TC & 56.8 & Perfect \\
\hline & MULSSR69R & GAAATGGTTTGCATCC & & & & & \\
\hline \multirow[t]{2}{*}{9} & M2SSR1F & CTCTCGAGAAAGCCATCA & GF107867 & 217 & CA & 50 & Perfect \\
\hline & M2SSR1R & GGTTGTCAAGTAGGACCG & & & & & \\
\hline \multirow[t]{2}{*}{10} & M2SSR5F & GCTCAGATTCGGTCATGG & GF109684 & 186 & TC & 50 & Perfect \\
\hline & M2SSR5R & CTGCTTCATGGTATCAGAGCAAGG & & & & & \\
\hline \multirow[t]{2}{*}{11} & M2SSR12F & GCGACCATTCAACAGAACCA & GF107890 & 270 & $A G$ & 50 & Perfect \\
\hline & M2SSR12R & GTGTTGTGGTTACTGGTTCC & & & & & \\
\hline \multirow[t]{2}{*}{12} & M2SSR13F & GTGTGTTGAGTGTAGCGGC & GF107891 & 154 & GT & 58 & Perfect \\
\hline & M2SSR13R & CGACGAAGATAACGACACGAC & & & & & \\
\hline \multirow[t]{2}{*}{13} & M2SSR19aF & GAAGAGCTCGCTACAAGG & GF107894 & 178 & TाTाC & 51.5 & Perfect \\
\hline & M2SSR19aR & GAAAGGCATGCTGCTCATG & & & & & \\
\hline \multirow[t]{2}{*}{14} & M2SSR2OF & CTAGAGAATCTTGGGCGATCC & GF107896 & 230 & TC & 55 & Perfect \\
\hline & M2SSR2OR & ACCGAGCGCTAGTTGTCAG & & & & & \\
\hline \multirow[t]{2}{*}{15} & M2SSR21F & GTTGCTGTGTGCTTGTGG & GF107897 & 247 & TG & 45 & Perfect \\
\hline & M2SSR21R & ACACAACACGTCAACCCAGA & & & & & \\
\hline \multirow[t]{2}{*}{16} & M2SSR53F & GTTGCTGAGCGTGGTGATAG & GF109658 & 172 & $A G$ & 50 & Perfect \\
\hline & M2SSR53R & ACGACACGCACACACGTC & & & & & \\
\hline \multirow[t]{2}{*}{17} & M2SSR65F & GGCTGATAATCGCAATGC & GF107874 & 173 & AGG & 51.5 & Perfect \\
\hline & M2SSR65R & GCGTGCCCACGTAGGAAG & & & & & \\
\hline \multirow[t]{2}{*}{18} & M2SSR67F & CGAGAAATTCCGACTCCATGGTC & GF107901 & 158 & $\mathrm{CTC}$ & 55 & Perfect \\
\hline & M2SSR67R & CCGGTGGTAGTGTTGCAAGAG & & & & & \\
\hline \multirow[t]{2}{*}{19} & M2SSR68F & AATTCCGACTCCATGGTCAG & GF107902 & 211 & TCT & 51.5 & Perfect \\
\hline & M2SSR68F & TTCCGGTGGTAGTGTTGC & & & & & \\
\hline \multirow[t]{2}{*}{20} & M2SSR93F & ATAGCCGATTTTGCAGGC & GF107877 & 243 & CTCC & 50 & Perfect \\
\hline & M2SSR93R & GAAATTCCGACTCCATGGTC & & & & & \\
\hline \multirow[t]{2}{*}{21} & M2SSR94bF & ATTAGCCGTGCATCTCTGG & GF107909 & 295 & ACTA & 55 & Perfect \\
\hline & M2SSR94bR & CGATCACTITCATGATCCGGG & & & & & \\
\hline \multirow[t]{2}{*}{22} & M2SSR102F & GAGCAAGGTTTCTGAACCC & GF107910 & 203 & AAG & 51.5 & Perfect \\
\hline & M2SSR102R & CTCAGCAGTCGTCTGAGG & & & & & \\
\hline
\end{tabular}


Table 2 Details of the genomic SSR markers developed for mulberry (Continued)

\begin{tabular}{|c|c|c|c|c|c|c|c|}
\hline \multirow[t]{2}{*}{23} & M2SSR121F & CGATCTGAAAGATGTCGTGC & GF107913 & 210 & CAC & 45 & Perfect \\
\hline & M2SSR121R & GCAACCGTCGTTCTCAGC & & & & & \\
\hline \multirow[t]{2}{*}{24} & Mul3SSR1F & CGGAAAGGGTCATGTTG & KF030980 & 150 & AAAT & 53 & Perfect \\
\hline & Mul3SSR1R & CTGTCGTTATTGAGAGAGCAGG & & & & & \\
\hline \multirow[t]{2}{*}{25} & Mul3SSR2F & GCTAGCAGATCCCACC & KF030981 & 261 & $C T$, GAGACC & 53 & Perfect \\
\hline & Mul3SSR2R & CAGCTCCTCTTCCACAAGC & & & & & \\
\hline \multirow[t]{2}{*}{26} & Mul3SSR4F & GGAGCAGTCAATCTCTTG & KF030982 & 314 & (ATATAC)CAC(TA) & 50 & interrupted \\
\hline & Mul3SSR4R & CTGGGGTTCAAACTAAGCTC & & & & & \\
\hline \multirow[t]{2}{*}{27} & Mul3SSR6F & GAGAGGTCGCCCCTTAG & KF030983 & 335 & GT & 51.5 & Perfect \\
\hline & Mul3SSR6R & GCCTCACAGGAGAACACC & & & & & \\
\hline \multirow[t]{2}{*}{28} & Mul3SSR7F & CCATGGCTCTTITGGTC & KF030984 & 198 & CTG & 48.5 & Perfect \\
\hline & Mul3SSR7R & GCAGAATCCAGCTIITTGG & & & & & \\
\hline \multirow[t]{2}{*}{29} & Mul3SSR9F & GACCAGCCATGAGCCTAC & KF030985 & 378 & $\mathrm{GT}, \mathrm{GA}$ & 51.5 & Compound \\
\hline & Mul3SSR9R & GGTTCACAACCACAATCTCC & & & & & \\
\hline \multirow[t]{2}{*}{30} & Mul3SSR14F & GGCGGTTAAGGAATATAGC & KF030986 & 227 & $A G$ & 47.5 & Perfect \\
\hline & Mul3SSR14R & CCAAAACGAGAAGAACG & & & & & \\
\hline \multirow[t]{2}{*}{31} & Mul3SSR16F & CTAGTAGCAGATCACCAC & KF030987 & 207 & A, AAAAG & 49.5 & Compound \\
\hline & Mul3SSR16R & CGGTCTCTCCCTAATCC & & & & & \\
\hline \multirow[t]{2}{*}{32} & Mul3SSR17 F & GTCTTGCACTAGGAGAGG & KF030988 & 345 & GT & 50.5 & Perfect \\
\hline & Mul3SSR17R & CTCACAGGAGAACACCACC & & & & & \\
\hline \multirow[t]{2}{*}{33} & Mul3SSR19F & CCAAGTCCTCCTCCAG & KF030989 & 172 & GAA & 50 & Perfect \\
\hline & Mul3SSR19R & GTTTGTGACTTGCCG & & & & & \\
\hline \multirow[t]{2}{*}{34} & Mul3SSR2OF & CTAGCAGATCGTGGCATTG & KF030990 & 252 & (CT)TTСTCTAT(CT) & 51 & interrupted \\
\hline & Mul3SSR20R & CTCCGCCCAAAATATCACAC & & & & & \\
\hline \multirow[t]{2}{*}{35} & Mul3SSR21F & CATCGCAAATAGGTGTGG & KF030991 & 239 & TC & 52.5 & Perfect \\
\hline & Mul3SSR21R & GGCAGTGAGAGCAAGGAG & & & & & \\
\hline \multirow[t]{2}{*}{36} & Mul3SSR23F & GCTAGCAGATCCCAAG & KF030992 & 224 & TGCCAC, TCT & 53.5 & Compound \\
\hline & Mul3SSR23R & CGAAACCCGCATTCATTC & & & & & \\
\hline \multirow[t]{2}{*}{37} & Mul3SSR24F & GCTCTTGTTGACACTGGC & KF030993 & 225 & TC & 51 & Perfect \\
\hline & Mul3SSR24R & CCGATTGTTTAAGGCC & & & & & \\
\hline \multirow[t]{2}{*}{38} & Mul3SSR25F & GAGCCTTGTTCACCAC & KF030994 & 155 & AAG & 50 & Perfect \\
\hline & Mul3SSR25R & GGTCAACTTTCATGCC & & & & & \\
\hline \multirow[t]{2}{*}{39} & Mul3SSR26F & GGTATGAGAGCTTCGCAC & KF030995 & 202 & $(\mathrm{TC}) \mathrm{G}(\mathrm{TC})$ & 52 & interrupted \\
\hline & Mul3SSR26R & GTCTCGGGAACAACAGC & & & & & \\
\hline \multirow[t]{2}{*}{40} & Mul3SSR28F & GGATCTTGCCATCTAGTGTG & KF030996 & 112 & $\mathrm{TA}, \mathrm{TG}$ & 53.5 & Compound \\
\hline & Mul3SSR28R & GCAGAATCATAGAGGACC & & & & & \\
\hline \multirow[t]{2}{*}{41} & Mul3SSR31F & GATCCACTTCCACTCCCAG & KF030997 & 382 & GTC, TTC & 52 & Compound \\
\hline & Mul3SSR31R & GGACGCATGAGGTITTAGG & & & & & \\
\hline \multirow[t]{2}{*}{42} & Mul3SSR33F & CTCCCGGATAAAAGACAACC & KF030998 & 390 & GAA & 48.5 & Perfect \\
\hline & Mul3SSR33R & CCTTGCTCATCATCATCG & & & & & \\
\hline \multirow[t]{2}{*}{43} & Mul3SSR34F & CATTITCCTCCTGACC & KF030999 & 221 & GA & 53 & Perfect \\
\hline & Mul3SSR34R & CAGTCCACGTCAGTTTC & & & & & \\
\hline \multirow[t]{2}{*}{44} & Mul3SSR36F & GCAGAATCCCGGAGAAGAG & KF031000 & 329 & GAA & 53 & Perfect \\
\hline & Mul3SSR36R & GCAGAATCCCCTGTTTGG & & & & & \\
\hline
\end{tabular}


Table 2 Details of the genomic SSR markers developed for mulberry (Continued)

\begin{tabular}{|c|c|c|c|c|c|c|c|}
\hline \multirow[t]{2}{*}{45} & Mul3SSR41F & CATCGCTCGTITTCGCATC & KF031001 & 251 & $\mathrm{CTT}$ & 49 & Perfect \\
\hline & Mul3SSR41R & CACTAGCCCCTGCACC & & & & & \\
\hline \multirow[t]{2}{*}{46} & Mul3SSR43F & CTCTGGAGTACAAGAACCG & KF031002 & 345 & GAA & 49.5 & Perfect \\
\hline & Mul3SSR43R & GGCACGATCCCAATCAAG & & & & & \\
\hline \multirow[t]{2}{*}{47} & Mul3SSR44F & CGCGTATTTCGGATTTCC & KF031003 & 238 & $C T, C A$ & 52 & Compound \\
\hline & Mul3SSR44R & GCTAGCAGAATCCCATC & & & & & \\
\hline \multirow[t]{2}{*}{48} & Mul3SSR49F & CAACATCAACACCGATCACC & KF031004 & 140 & TCA & 52 & Perfect \\
\hline & Mul3SSR49R & GCAGAATCCCACCAACATC & & & & & \\
\hline \multirow[t]{2}{*}{49} & Mul3SSR50F & CTAGCAGATCCACCAAACC & KF031005 & 161 & $\mathrm{CTT}$ & 53 & Perfect \\
\hline & Mul3SSR50R & GTTGTTGTACTCTCGCACG & & & & & \\
\hline \multirow[t]{2}{*}{50} & Mul3SSR52F & CAGATCCCATACACAAAGCC & KF031006 & 391 & TाTाTC & 51.5 & interrupted \\
\hline & Mul3SSR52R & GTGAGAGAACCCGAGAAG & & & & & \\
\hline \multirow[t]{2}{*}{51} & Mul3SSR53F & CAGCTATGACCATGATTACGCC & KF031007 & 124 & AAAAC & 50.5 & Perfect \\
\hline & Mul3SSR53R & GGACCCTTGATGGCATTG & & & & & \\
\hline \multirow[t]{2}{*}{52} & Mul3SSR64F & GACGAAAACCGATGAAGAGG & KC408230 & 380 & ATGAGC & 47.9 & Perfect \\
\hline & Mul3SSR64R & GACCGGTAAAACCACACACC & & & & & \\
\hline \multirow[t]{2}{*}{53} & Mul3SSR65F & CTGGAGTACAAGAACCGCAAC & KC408231 & 220 & GAA & 53.8 & Perfect \\
\hline & Mul3SSR65R & GCCCTCCACCATTGAACTAAG & & & & & \\
\hline \multirow[t]{2}{*}{54} & Mul3SSR66F & GCGAATGATGAAAACGGAGAGG & KC408232 & 262 & TाTA & 52.8 & Perfect \\
\hline & Mul3SSR66R & GCGGTTAGTTGCCTAGTTGG & & & & & \\
\hline \multirow[t]{2}{*}{55} & Mul3SSR67F & ATACCACGTTCCGGTGTG & KC408233 & 304 & GT, GA & 52.8 & Compound \\
\hline & Mul3SSR67R & CATACCGTGCCCCAACTTAC & & & & & \\
\hline \multirow[t]{2}{*}{56} & Mul3SSR70F & GAAGAGGGGAGAGGGAGAGA & KC408236 & 187 & AAATAA & 54.1 & Perfect \\
\hline & Mul3SSR70R & CAACCAGGATCCAAATAGAAGC & & & & & \\
\hline \multirow[t]{2}{*}{57} & Mul3SSR71F & GGATACTACCTGTTTGGTTGCTG & KC408237 & 360 & AAAT, GAA & 54.5 & Compound \\
\hline & Mul3SSR71R & ATTCCСTCCTCAACGAC & & & & & \\
\hline \multirow[t]{2}{*}{58} & Mul3SSR72F & CATCCTTCGAATCCAAGAGC & KC408238 & 231 & (AG)TTAACCCAAAGAAT(AG) & 50.8 & interrupted \\
\hline & Mul3SSR72R & CGAGAGGAAATCCTCACAGC & & & & & \\
\hline \multirow[t]{2}{*}{59} & Mul3SSR73F & GGGGAGGTAGCTGATGTGTC & KC408239 & 318 & TA, TATT & 49.1 & Compound \\
\hline & Mul3SSR73R & AGCATGCCCTTCCATATCAC & & & & & \\
\hline \multirow[t]{2}{*}{60} & Mul3SSR74F & CCCATTGAGGGTTITGTGAG & KC408240 & 407 & AG, GTGAGC & 54.8 & Compound \\
\hline & Mul3SSR74R & ATGTGAGCTCGGGATTTGAC & & & & & \\
\hline \multirow[t]{2}{*}{61} & Mul3SSR75F & CAGGTTGAACGCCCATTACTC & KC408241 & 102 & $C T, T C A, T C$ & 47.9 & compound \\
\hline & Mul3SSR75R & GTGCAGAATGTCAGTATGCG & & & & & \\
\hline \multirow[t]{2}{*}{62} & Mul3SSR77F & ACTCCGCCTGAAGAACGAAG & KC408243 & 254 & AGA & 54.8 & Perfect \\
\hline & Mul3SSR77R & TAGCAGAATCCCCTGTTTGG & & & & & \\
\hline \multirow[t]{2}{*}{63} & Mul3SSR80F & GAGCCGTTTGATTTCCGTC & KC408245 & 158 & CT & 47.9 & Perfect \\
\hline & Mul3SSR80R & CAACGGTCGGTGAAAAAGC & & & & & \\
\hline \multirow[t]{2}{*}{64} & Mul3SSR91F & CATGAACCGTTGGATCACAG & KC408246 & 277 & $A G$ & 54.8 & Perfect \\
\hline & Mul3SSR91R & ATCCCAGATCCCAAATACCC & & & & & \\
\hline \multirow[t]{2}{*}{65} & Mul3SSR93F & CAGCCAATGCACTITTAACG & KC408248 & 343 & $A C$ & 49.1 & Perfect \\
\hline & Mul3SSR93R & GTGGAGCTTCTGTTGAGC & & & & & \\
\hline \multirow[t]{2}{*}{66} & Mul3SSR94F & CCCTCATGTGTTCCATCTACC & KC408249 & 198 & AAAACAA & 52.8 & perfect \\
\hline & Mul3SSR94R & CAGAATCACAGCCGAGGAAG & & & & & \\
\hline
\end{tabular}


Table 2 Details of the genomic SSR markers developed for mulberry (Continued)

\begin{tabular}{|c|c|c|c|c|c|c|c|}
\hline \multirow[t]{2}{*}{67} & Mul3SSR95F & GATCATCGTGCCAATAAGCC & KC408250 & 209 & $A G$ & 52.8 & perfect \\
\hline & Mul3SSR95R & TAAGAGCTGAGAGGGGAAGC & & & & & \\
\hline \multirow[t]{2}{*}{68} & Mul3SSR97F & TCCACCACTGAACCAAATC & KC408358 & 292 & GAA & 50.8 & Perfect \\
\hline & Mul3SSR97R & ATTAGGGTTGTGACGACGAC & & & & & \\
\hline \multirow[t]{2}{*}{69} & Mul3SSR98F & ACGACAATGCTGTCGTCTTG & KC408252 & 286 & TG & 55.2 & Perfect \\
\hline & Mul3SSR98R & CGATTCGGAAAGCAAACCAAAC & & & & & \\
\hline \multirow[t]{2}{*}{70} & Mul3SSR99F & AGGCAAAGGAGCAGGATG & KC408253 & 272 & TTC & 58.5 & perfect \\
\hline & Mul3SSR99R & GTGGTCACTGCAAAAAGC & & & & & \\
\hline \multirow[t]{2}{*}{71} & Mul3SSR101F & TGAGCCAAGACAAGGAGACA & KC408255 & 330 & $A C$ & 50.8 & Perfect \\
\hline & Mul3SSR101R & AGCTAGCAGAATCCCCTTGA & & & & & \\
\hline \multirow[t]{2}{*}{72} & Mul3SSR102F & TTGGTTGCTGAGAAATGCAG & KC408256 & 230 & AAAT, GAA & 55.4 & Compound \\
\hline & Mul3SSR102R & TTGTCGATGGAAAACACGAC & & & & & \\
\hline \multirow[t]{2}{*}{73} & Mul3SSR103F & GGTCAGATCAGTTTCGTTGC & KC408257 & 258 & $A G$ & 53.3 & Perfect \\
\hline & Mul3SSR103R & GTAAGAGCTGAGAGGGGAAG & & & & & \\
\hline \multirow[t]{2}{*}{74} & Mul3SSR104F & GAAGAGCCGACAAAGAATGG & KC408258 & 225 & ATGAGC, GCAGAGAA & 53.3 & Compound \\
\hline & Mul3SSR104R & GGAATGCTTGACCTITGACC & & & & & \\
\hline \multirow[t]{2}{*}{75} & Mul3SSR105F & GCAGAATCCCAAGTTAATGCC & KC408259 & 254 & TCT, TGCCAC & 57.1 & Compound \\
\hline & Mul3SSR105R & CCTCATAGAGTACAGGAACCG & & & & & \\
\hline \multirow[t]{2}{*}{76} & Mul3SSR108F & TCTGCCATGGATGCGTGC & KC408262 & 215 & CCTCT, TC, TC & 54.1 & Compound \\
\hline & Mul3SSR108R & GACAGAAACCCGGCAGAAG & & & & & \\
\hline \multirow[t]{2}{*}{77} & Mul3SSR114F & GCAACTCTGCCTTGTTTTC & KC408266 & 106 & $A G$ & 58.5 & Perfect \\
\hline & Mul3SSR114R & TGGTGCCTTAGACCAGAC & & & & & \\
\hline \multirow[t]{2}{*}{78} & Mul3SSR116F & GATTITCAGCGCATGGTTC & KC408267 & 382 & TाTA, AATA & 58.5 & Compound \\
\hline & Mul3SSR116R & CCAAGGAAGGTGAAATCC & & & & & \\
\hline \multirow[t]{2}{*}{79} & Mul3SSR118F & CATGAACCGTTGGATCACAG & KC408269 & 277 & $A G$ & 53.3 & Perfect \\
\hline & Mul3SSR118R & ATCCCAGATCCCAAATACCC & & & & & \\
\hline \multirow[t]{2}{*}{80} & Mul3SSR122F & GGTGATGGGCTIITGATG & KC408273 & 219 & ATC & 51.7 & Perfect \\
\hline & Mul3SSR122R & GTTGGATCTGAGGAGGGTC & & & & & \\
\hline \multirow[t]{2}{*}{81} & Mul3SSR124F & GGGTGCCAAGGAAAGGA & KC408275 & 228 & TCTTTC & 54.8 & Perfect \\
\hline & Mul3SSR124R & AGAGAGATTCGGCAAAACC & & & & & \\
\hline \multirow[t]{2}{*}{82} & Mul3SSR125F & CTITGATGATGCTTCCTCTGC & KC408276 & 261 & $\mathrm{CTT}, \mathrm{CTA}$ & 54.1 & Compound \\
\hline & Mul3SSR125R & GTGCACGGAATTTGCTACTG & & & & & \\
\hline \multirow[t]{2}{*}{83} & Mul3SSR126F & GGATGCTATTGCCTAAAGTG & KC408277 & 199 & AAAAG, AAAAGA & 52.8 & Compound \\
\hline & Mul3SSR126R & GCAGAATCAGAAGTGTTGTCC & & & & & \\
\hline \multirow[t]{2}{*}{84} & Mul3SSR127F & CGATTGCCACATGTTCAGAC & KC408278 & 309 & $A C$ & 52.8 & Perfect \\
\hline & Mul3SSR127R & GGCAGACCCGATAAGCAGTA & & & & & \\
\hline \multirow[t]{2}{*}{85} & Mul3SSR131F & ACTGTGCTTCGTGGAGTTG & KC408279 & 305 & $\mathrm{CT}, \mathrm{TCA}$ & 55.4 & Compound \\
\hline & Mul3SSR131R & GAGAGCTTCGAGAGGGAGG & & & & & \\
\hline \multirow[t]{2}{*}{86} & Mul3SSR135F & GATCATCACAAAAAGGCTGG & KC408282 & 137 & TC & 55.4 & Perfect \\
\hline & Mul3SSR135R & GATTGCCGACACTCGTATC & & & & & \\
\hline \multirow[t]{2}{*}{87} & Mul3SSR141F & TTGGTGCACTTGCCAAAC & KC408286 & 336 & TTGTT, T & 52.8 & Compound \\
\hline & Mul3SSR141R & TCACCTCGCATAGACCAC & & & & & \\
\hline \multirow[t]{2}{*}{88} & Mul3SSR142F & GCAGAATCCCAAACTTGAGAG & KC408287 & 213 & (AG)AAGCTGAAAATGGGGTGT(AG) & 54.5 & interrupted \\
\hline & Mul3SSR142R & CACAGTTAGCATCACCATGTC & & & & & \\
\hline
\end{tabular}


Table 2 Details of the genomic SSR markers developed for mulberry (Continued)

\begin{tabular}{|c|c|c|c|c|c|c|c|}
\hline \multirow[t]{2}{*}{89} & Mul3SSR143F & TGCCACCTTCTCCAATATG & KC408288 & 151 & TTA & 54.5 & Perfect \\
\hline & Mul3SSR143R & CGGGAATCGGGATTAAG & & & & & \\
\hline \multirow[t]{2}{*}{90} & Mul3SSR144F & GATATGGGAACAAGGGCACTG & KC408289 & 284 & CATCAC, ACT & 54.5 & Compound \\
\hline & Mul3SSR144R & CTGTTGATGAAGCCATGATG & & & & & \\
\hline \multirow[t]{2}{*}{91} & Mul3SSR145F & ССТTСТTCСССАТАСССАC & KC408290 & 165 & TCA & 50.4 & perfect \\
\hline & Mul3SSR145R & CATTTCGGAAGCTTGTCCA & & & & & \\
\hline \multirow[t]{2}{*}{92} & Mul3SSR146F & CAACCGATTACATGGTGTGG & KC408291 & 256 & CT & 50.4 & perfect \\
\hline & Mul3SSR146R & TTCCGCAGCAAGCTTTAC & & & & & \\
\hline \multirow[t]{2}{*}{93} & Mul3SSR148F & AGGCAATGACAAACGGAAG & KC408293 & 156 & CAA & 45.1 & Perfect \\
\hline & Mul3SSR148R & GCAACCACTTCTGTGTGAGC & & & & & \\
\hline \multirow[t]{2}{*}{94} & Mul3SSR149F & TGTCTCTTGGTCAGCGTCTC & KC408294 & 280 & (AC)TATACATTCGT(AC) & 54.8 & interrupted \\
\hline & Mul3SSR149R & CATTTCCCAGAAAGCCACTTC & & & & & \\
\hline \multirow[t]{2}{*}{95} & Mul3SSR150F & TCCTGTCTTAGATCGCAACG & KC408295 & 226 & TTTA, AAG & 54.8 & Compound \\
\hline & Mul3SSR150R & GGTGGCAGGGATTAATGAG & & & & & \\
\hline \multirow[t]{2}{*}{96} & Mul3SSR151F & GAGTITGCAGCCTCAGTATGG & KC408296 & 196 & $\mathrm{GT}, \mathrm{T}$ & 54.8 & Compound \\
\hline & Mul3SSR151R & CGTGCTTGGAGTAAGGGAAG & & & & & \\
\hline \multirow[t]{2}{*}{97} & Mul3SSR152F & TCTCTGTCTGCGCATCAATC & KC408297 & 189 & TC & 54.5 & Perfect \\
\hline & Mul3SSR152R & GCAGAATCCCGATTTTACAG & & & & & \\
\hline \multirow[t]{2}{*}{98} & Mul3SSR153F & GGGCATTGTATTGTCCAAGC & KC408298 & 302 & TTA & 51.7 & Perfect \\
\hline & Mul3SSR153R & GAGTAGCCGACATAAATCAGC & & & & & \\
\hline \multirow[t]{2}{*}{99} & Mul3SSR155F & ACCCTAAATTGGGACGGAAG & KC408300 & 105 & AAG & 54.5 & Perfect \\
\hline & Mul3SSR155R & CGATTTCTACGAATGCCAGAC & & & & & \\
\hline \multirow[t]{2}{*}{100} & Mul3SSR156F & CCCACCCAATCACAATAACC & KC408301 & 190 & GAA & & Perfect \\
\hline & Mul3SSR156R & GTCAACTCCCGAGCTCAC & & & & & \\
\hline \multirow[t]{2}{*}{101} & Mul3SSR159F & CCCAGTTGGGGTTGAGTTG & KC408304 & 108 & $\pi \mathrm{TC}$ & 51.7 & Perfect \\
\hline & Mul3SSR159R & CCTGTCTTGGAGAGGAGAAC & & & & & \\
\hline \multirow[t]{2}{*}{102} & Mul3SSR160F & СССTCTCTCTCGTCGTTCTC & KC408305 & 171 & $\mathrm{CT}$ & 54.8 & Perfect \\
\hline & Mul3SSR160R & CCCACTCAACCCGTTTATG & & & & & \\
\hline \multirow[t]{2}{*}{103} & Mul3SSR161F & TGCATGTACTGGATGATGTG & KC408306 & 166 & TGAAG & 54.8 & Perfect \\
\hline & Mul3SSR161R & CTTGGCTGTAGAAGCACG & & & & & \\
\hline \multirow[t]{2}{*}{104} & Mul3SSR163F & CAGATCTTCTCTCTTGCTCC & KC408308 & 221 & $C T, C A$ & 54.5 & Compound \\
\hline & Mul3SSR163R & GTATGTTTGCTTCACGGCTC & & & & & \\
\hline \multirow[t]{2}{*}{105} & Mul3SSR164F & CGGCGGTGGAGAAACAAAG & KC408309 & 393 & GA, AAAG, AAAAAG & 54.8 & Compound \\
\hline & Mul3SSR164R & GTGAACCCCTGTCTTGGATG & & & & & \\
\hline \multirow[t]{2}{*}{106} & Mul3SSR166F & AAGAGAACAGTGGCCGTC & KC408311 & 222 & ATCACC & 54.8 & Perfect \\
\hline & Mul3SSR166R & AGGGAAAGGCAAGACTAGGG & & & & & \\
\hline \multirow[t]{2}{*}{107} & Mul3SSR167F & ССТTCTTCCCCATACCCAC & KC408312 & 190 & TCA & 49.1 & Perfect \\
\hline & Mul3SSR167R & CACATTTCGGAAGCTTGTCC & & & & & \\
\hline \multirow[t]{2}{*}{108} & Mul3SSR168F & СCCTTTAATCCTCTGCCTG & KC408313 & 267 & $A C$ & 50.4 & Perfect \\
\hline & Mul3SSR168R & GCTGATACTTGGGGTTGG & & & & & \\
\hline \multirow[t]{2}{*}{109} & Mul3SSR169F & CCAGTTGGGGTTGAGTTGTAAC & KC408314 & 107 & TTC & 54.8 & Perfect \\
\hline & Mul3SSR169R & CCTGTCTTGGAGAGGAGAACC & & & & & \\
\hline \multirow[t]{2}{*}{110} & Mul3SSR170F & TAGCTAGCAGATCCCTAC & KC408315 & 241 & GT & 49.1 & Perfect \\
\hline & Mul3SSR170R & GGATTTCGTCGCAACCAT & & & & & \\
\hline
\end{tabular}


Table 2 Details of the genomic SSR markers developed for mulberry (Continued)

\begin{tabular}{|c|c|c|c|c|c|c|c|}
\hline \multirow[t]{2}{*}{111} & Mul3SSR171F & GGAGGGGTTTTCCTTGAC & KC408316 & 168 & GAA & 51.7 & Perfect \\
\hline & Mul3SSR171R & CGAAGTGGTGCTCTTCAC & & & & & \\
\hline \multirow[t]{2}{*}{112} & Mul3SSR172F & GCTAGGCTAAAGCCTGGAAG & KC408317 & 140 & TGGATA & 54.5 & Perfect \\
\hline & Mul3SSR172R & TAGTTCCGGTGACCAACTCC & & & & & \\
\hline \multirow[t]{2}{*}{113} & Mul3SSR173F & TCCCGGAACAATCTTATGG & KC408318 & 304 & $\mathrm{CTT}, \mathrm{CTA}$ & 54.5 & Compound \\
\hline & Mul3SSR173R & CCCTAGTGCACCTTCATTTC & & & & & \\
\hline \multirow[t]{2}{*}{114} & Mul3SSR174F & AGCGGTTCTTGTGAGCAG & KC408319 & 371 & $\mathrm{~A}, \mathrm{TTC}$ & 54.8 & Perfect \\
\hline & Mul3SSR174R & CATAGTTTGGGCCCGTTTAG & & & & & \\
\hline \multirow[t]{2}{*}{115} & Mul3SSR175F & GGAAAAGAAAGGGGGAATCAG & KC408320 & 127 & GT & 54.8 & Perfect \\
\hline & Mul3SSR175R & GTCTCCTIITGGGGATACCA & & & & & \\
\hline \multirow[t]{2}{*}{116} & Mul3SSR177F & CACGTACGCAACTITTTCC & KC408322 & 329 & $A G$ & 49.1 & Perfect \\
\hline & Mul3SSR177R & GTGAGGCTTGACCTGAATG & & & & & \\
\hline \multirow[t]{2}{*}{117} & Mul3SSR178F & CAGAGGAGGATATGACATTATCAAC & KC408323 & 202 & TC & 49.1 & Perfect \\
\hline & Mul3SSR178R & CAAACAGAATCCCACACACG & & & & & \\
\hline \multirow[t]{2}{*}{118} & Mul3SSR179F & CCAGTTGGGGTTGAGTTGTAAC & KC408324 & 107 & TTC & 50.4 & Perfect \\
\hline & Mul3SSR179R & CCTGTCTTGGAGAGGAGAACC & & & & & \\
\hline \multirow[t]{2}{*}{119} & Mul3SSR180F & TCGCCACAATCTTTCACTTG & KC408325 & 335 & TCA, TCT & 54.8 & Compound \\
\hline & Mul3SSR180R & GCGGAGGAATTITCCATC & & & & & \\
\hline \multirow[t]{2}{*}{120} & Mul3SSR181F & CTCTGACATTGGCAAGAAAGC & KC408326 & 282 & TTC & 51.7 & Perfect \\
\hline & Mul3SSR181R & GAGGAACGGCAATAAGAGG & & & & & \\
\hline \multirow[t]{2}{*}{121} & Mul3SSR183F & GATCAGGAGAGGAAGGAG & $J X 258829$ & 150 & AGA & 52.8 & Perfect \\
\hline & Mul3SSR183R & CTGTCAAAACCAGCCTTG & & & & & \\
\hline \multirow[t]{2}{*}{122} & Mul3SSR184F & CATTCCTGGTGTCAGCCT & $J X 258830$ & 163 & $(\mathrm{TC}) \mathrm{T}(\mathrm{TC})$ & 51.7 & interrupted \\
\hline & Mul3SSR184R & CAGATCGGCACCAATAGT & & & & & \\
\hline \multirow[t]{2}{*}{123} & Mul3SSR185F & AGAGAGCAACCACGGGAAG & $J X 465665$ & 336 & AAAAAG & 52.8 & Perfect \\
\hline & Mul3SSR185R & GTGAACCCCTGTCTTGGA & & & & & \\
\hline \multirow[t]{2}{*}{124} & Mul3SSR187F & GGACATTTCACAACCCTG & JX465667 & 324 & AAT, CT, AGA & 53.8 & Compound \\
\hline & Mul3SSR187R & AACTGCAAGTTGGCACAG & & & & & \\
\hline \multirow[t]{2}{*}{125} & Mul3SSR190F & AGCTGGGTGGAGGATTG & $J X 465669$ & 283 & AC, GCAC & 54.8 & Compound \\
\hline & Mul3SSR190R & CCACCTCTGCAAGGATTG & & & & & \\
\hline \multirow[t]{2}{*}{126} & Mul3SSR191F & CGAATGCATAGAGGGAGAGC & $J X 465670$ & 386 & AAAAC & 50.4 & Perfect \\
\hline & Mul3SSR191R & CACTTGAGGGTTCATTCAGC & & & & & \\
\hline \multirow[t]{2}{*}{127} & Mul3SSR192F & GACCTACTTCTCGAACAGTAAC & JX465671 & 198 & AAAAC & 54.8 & Perfect \\
\hline & Mul3SSR192R & CTTGAGGGTTCATTCAGC & & & & & \\
\hline \multirow[t]{2}{*}{128} & Mul3SSR193F & GCTAGTTCCATCGCCCATAG & JX465672 & 358 & TTGA, TG & 51.7 & Compound \\
\hline & Mul3SSR193R & GCATCAGATAAAGCAGGTG & & & & & \\
\hline \multirow[t]{2}{*}{129} & Mul3SSR197F & GGTGAAAGTTCGTGTGAGTCC & JX465674 & 186 & TCT, TC & 54.8 & Compound \\
\hline & Mul3SSR197R & TCAGCAACTAGAGTGACTITG & & & & & \\
\hline \multirow[t]{2}{*}{130} & Mul3SSR199F & CTCAGGTACGCTGTGCTG & JX465675 & 238 & TC & 54.8 & Perfect \\
\hline & Mul3SSR199R & GACTCAAAGCACATGCCAAG & & & & & \\
\hline \multirow[t]{2}{*}{131} & Mul3SSR201F & CCATTGAGGGTITTGTGAG & JX465677 & 406 & GA, GTGAGC & 54.8 & Compound \\
\hline & Mul3SSR201R & ATGTGAGCTCGGGATTTGAC & & & & & \\
\hline \multirow[t]{2}{*}{132} & Mul3SSR202F & CCCTCTCGATCATCACC & KC408332 & 230 & TTC & 49.1 & Compound \\
\hline & Mul3SSR202R & CGGAGACGTAGATGCCC & & & & & \\
\hline
\end{tabular}


Table 2 Details of the genomic SSR markers developed for mulberry (Continued)

\begin{tabular}{|c|c|c|c|c|c|c|c|}
\hline \multirow[t]{2}{*}{133} & Mul3SSR203F & GACCGTAGGAGAGAGTGC & KC408333 & 442 & $\mathrm{~T}, \mathrm{G}, \mathrm{CG}$ & 54.8 & Compound \\
\hline & Mul3SSR203R & GGATACCCGCTAAACCCAC & & & & & \\
\hline \multirow[t]{2}{*}{134} & Mul3SSR205F & GCAGTTCCGAATCACGAAATAGG & KC408335 & 216 & TTA & 49.1 & Perfect \\
\hline & Mul3SSR205R & CAAGGCGAGGTAAACACC & & & & & \\
\hline \multirow[t]{2}{*}{135} & Mul3SSR214F & GTGGAACAGGGAGCCAGTCT & KC408344 & 297 & GGGCG, GAG, GAGGA & 54.8 & Compound \\
\hline & Mul3SSR214R & CATGCACGTCTCACTCCAC & & & & & \\
\hline \multirow[t]{2}{*}{136} & Mul3SSR229F & CCTTATAGCCGATITIGCAGGC & KC408354 & 247 & $\mathrm{TCT}$ & 54.8 & Perfect \\
\hline & Mul3SSR229R & GAAATTCCGACTCCATGGTC & & & & & \\
\hline \multirow[t]{2}{*}{137} & Mul3SSR230F & CGGGTGAGCTGGTTTGTTC & KC408355 & 298 & GT, TG & 50.4 & Compound \\
\hline & Mul3SSR230R & CAGCCCCACAATCCCTACT & & & & & \\
\hline
\end{tabular}

detected 232 microsatellite motifs that could be classified into interrupted and compound repeat types (Table 2). Of these repeat types, $86(37.1 \%)$ were DNR, 73 (31.5\%) TNR, 19 (8.2\%) TtNR, 27 (11.6\%) PNR and 27 (11.6\%) were HNR types (Figure 1). These genomic SSR markers developed for mulberry have been deposited in the NCBI GenBank database and the details of all the locus specific primers are given in Table 2.

\section{Isolation and characterization of genic microsatellites}

A set of 2391 stress specific EST sequences obtained by subjecting K2, a leading mulberry variety [19-21], was examined for the presence of repeat motifs and 800 sequences were found to contain a total of 1155 genic microsatellite regions (Table 1). Of these, 254 sequences were found to contain more than one microsatellite locus. Mono nucleotide repeats were the most common among the sequences (Figure 1) followed by tri and hexa-repeat motifs (28.3\% and $38.3 \%$ respectively). Among the factors that cause the generation of repeat sequences in the genome, replication slippage is often considered as the major mechanism. Though, this is a random phenomenon, the slippage in genic regions occurs in repeats of three bases clubbed with frame shift mutations which suppresses non-triplet repeats resulting in the abundance of TNR and HNR motifs [44-46]. A total of 180 compatible microsatellite regions were identified represented by 136 primer pairs (Figure 1). A significant $87.5 \%$ of these were perfect while $5.8 \%$ were interrupted and $6.6 \%$ were compound repeats (Table 3 ).

It appears that the forces causing tandem repeats such as mutation, replication slippage etc., occurred more frequently in non-coding regions than the genic regions $[22,45,47]$. It is also possible that the lethal mutations in genic regions would subsequently eliminate the genotype while the sequence variations in non-coding regions of the genome would persist, resulting in the observation of higher frequency of sequence variations in the noncoding genomic regions. Accordingly, more numbers of repeat regions were found on the genomic regions (82\%) while $48 \%$ were found in the genic regions. A large number of clones with more than $15 \mathrm{bp}$ of repeat motifs were found among the markers developed. Results revealed that the frequency of such markers was more in the non-coding regions of the mulberry genome than the genic regions [25]. The presence of longer repeats in the genome may have an evolutionary advantage leading to differences in the ability to adapt to new environments $[48,49]$.

\section{Validation of genomic and genic SSR markers}

The genic and genomic SSR markers were validated using four contrasting genotypes of Morus alba that were chosen based on variations in certain physiological traits [50] and seven different mulberry species (all belonging to the genus Morus) (Table 4). Of the 222 genomic and 136 genic SSR markers screened, 137 (62\%) genomic and 51 (37\%) genic SSR markers showed single locus amplification in all the Morus species as well as genotypes of Morus alba (Table 5). Further, genomic SSRs exhibited greater levels of polymorphism compared with the genic SSR markers. Such phenomenon has also been reported in other plant species [51]. Of the 188 markers examined, $87(46.2 \%)$ detected heterozygosity in the mulberry genotypes and species with a maximum of 1.00 for markers MulSSR39, Mul3SSR26 Mul3SSR91 and Mul3SSR135, (Additional file 1). Around 41\% of the genic markers also detected heterozygosity among the mulberry genotypes and species (Additional file 1). SSR markers are highly suited for mapping even in cross pollinated species because of their ability to detect heterozygosity. The markers developed in this study also detected significant levels of heterozygosity in mulberry species and genotypes.

Variations in the genic regions, though less frequent, would have a greater possibility of having a direct role in altering the phenotype of an organism [52]. The variability obtained for the SSR markers across mulberry species and genotypes was analyzed using Power Marker version 3.25 and the results are summarized in Table 6. A total 
Table 3 Details of the genic (EST) SSR markers developed for mulberry

\begin{tabular}{|c|c|c|c|c|c|c|c|}
\hline SI no & Primer name & Primer sequence & GenBank-ID & Amplicon size & Repeat motif & $\mathrm{Ta}\left(0^{\circ} \mathrm{C}\right)$ & Repeat type \\
\hline \multirow[t]{2}{*}{1} & MESTSSR10F & CATTGCACATTGCAGGTAGC & GT629469.1 & 237 & GTT & 52.8 & Perfect \\
\hline & MESTSSR10R & CGGCCATCCAAAATGTTGTTC & & & & & \\
\hline \multirow[t]{2}{*}{2} & MESTSSR13F & TCTATCTCAACCGGAAGTCC & GT628644.1 & 230 & (CAAAAG)G(AAAATA) & 54.8 & interrupted \\
\hline & MESTSSR13R & CCAATTTGCTCGTCTTATGC & & & & & \\
\hline \multirow[t]{2}{*}{3} & MESTSSR14F & CGGCCACAGGTACTTTC & GT628768.1 & 202 & TTGATT & 50.4 & Perfect \\
\hline & MESTSSR14R & GGCAGCGATTTAGGATTGG & & & & & \\
\hline \multirow[t]{2}{*}{4} & MESTSSR2OF & CGCAAGTGTCTCAACTG & GT629110.1 & 200 & TGA & 49.1 & Perfect \\
\hline & MESTSSR2OR & GGAACGGATGGAGTAAG & & & & & \\
\hline \multirow[t]{2}{*}{5} & MESTSSR23F & GGCCCAAACTCCATAGC & ES448350.1 & 202 & TAC & 50.4 & Perfect \\
\hline & MESTSSR23R & CCGCCAATTCTAGACCAATG & & & & & \\
\hline \multirow[t]{2}{*}{6} & MESTSSR26F & CGTGATTACCTTCGGATTGG & ES448391.1 & 219 & AGCTGG & 57.9 & Perfect \\
\hline & MESTSSR26R & CCAACCCAGTAGACCCAGTG & & & & & \\
\hline \multirow[t]{2}{*}{7} & MESTSSR27F & CCAACATTATCCGGAACACC & ES448394.1 & 266 & CGG & 54.8 & Perfect \\
\hline & MESTSSR27R & GGTAAAGCCATCCGTTGC & & & & & \\
\hline \multirow[t]{2}{*}{8} & MESTSSR28F & GCCCAGTITCCCACAGAA & ES448403.1 & 217 & ATA & 47.9 & Perfect \\
\hline & MESTSSR28R & GGATGGTTTGTGCGTGC & & & & & \\
\hline \multirow[t]{2}{*}{9} & MESTSSR31F & CACCAATTAAAAGCGCAGTG & ES448813.1 & 204 & GA & 57.9 & Perfect \\
\hline & MESTSSR31R & CTITGTGGTTGGCTCGTG & & & & & \\
\hline \multirow[t]{2}{*}{10} & MESTSSR35F & CGTTITCCGCTTCAGAGAG & ES448478.1 & 206 & $A G$ & 54.8 & Perfect \\
\hline & MESTSSR35R & GCCGATATCCTCCTTTCCTC & & & & & \\
\hline \multirow[t]{2}{*}{11} & MESTSSR37F & CAAAAGCGGTTGGAATAGC & ES448476.1 & 245 & (CTTTC) CTCC(T) & 54.8 & interrupted \\
\hline & MESTSSR37R & CCTCAACACAAAACCCACC & & & & & \\
\hline \multirow[t]{2}{*}{12} & MESTSSR40F & GAATCCTACAAGGGAGC & ES449069.1 & 215 & AAAAT & 52.8 & Perfect \\
\hline & MESTSSR40R & CATACAAGGATGCCCACC & & & & & \\
\hline \multirow[t]{2}{*}{13} & MESTSSR41F & GGTCGACAAGAGGTAATC & ES449022.1 & 121 & AAAAG & 56.7 & Perfect \\
\hline & MESTSSR41R & GAAGGCACCGAAGAGAAC & & & & & \\
\hline \multirow[t]{2}{*}{14} & MESTSSR42F & CAAGAGGTAATCCGTTC & ES448502.1 & 254 & AG & 54.8 & Perfect \\
\hline & MESTSSR42R & CGTTGTTAGCAGGAGC & & & & & \\
\hline \multirow[t]{2}{*}{15} & MESTSSR46F & GCCCATGTTTGCGGAG & ES449184.1 & 200 & $A G$ & 56.7 & Perfect \\
\hline & MESTSSR46R & GGATTITCTGTCTGGGTG & & & & & \\
\hline \multirow[t]{2}{*}{16} & MESTSSR47F & GACTGCGGGAGAACAG & ES448510.1 & 220 & $\mathrm{CTC}$ & 54.8 & Perfect \\
\hline & MESTSSR47R & GTTCACCGAGGCTGAGAG & & & & & \\
\hline \multirow[t]{2}{*}{17} & MESTSSR48F & GTTGTGGTGGTTGTTGC & ES448516.1 & 201 & $\mathrm{TC}$ & 56.7 & Perfect \\
\hline & MESTSSR48R & ССTTCACTTTCTCGCC & & & & & \\
\hline \multirow[t]{2}{*}{18} & MESTSSR49F & CTTCGACGCCTTCTGCG & ES448598.1 & 184 & GAAGA & 56.7 & Perfect \\
\hline & MESTSSR49R & GAGCGTCTCGAAGCAGTTG & & & & & \\
\hline \multirow[t]{2}{*}{19} & MESTSSR50F & GCCGGCATGTACGGATA & ES448967.1 & 235 & CCTAAC & 54.8 & Perfect \\
\hline & MESTSSR50R & GTAAAAGTTTCGCCCCAGG & & & & & \\
\hline \multirow[t]{2}{*}{20} & MESTSSR51F & CCTAGGGTTTCCTTCGCTTC & ES448621.1 & 223 & GCG & 54.8 & Perfect \\
\hline & MESTSSR51R & CGCTTAGGCTCCTTCCTC & & & & & \\
\hline \multirow[t]{2}{*}{21} & MESTSSR52F & CTTCGTTACGCTCGCTATG & ES448640.1 & 261 & TATTाT & 56.7 & Perfect \\
\hline & MESTSSR52R & CCTTCTCTCAAGAATACTGG & & & & & \\
\hline \multirow[t]{2}{*}{22} & MESTSSR53F & GGCCAACATGTACGGATAG & ES449078.1 & 203 & CCTAAC & 56.7 & Perfect \\
\hline & MESTSSR53R & CGCCAGGTACAACAAGAAG & & & & & \\
\hline
\end{tabular}


Table 3 Details of the genic (EST) SSR markers developed for mulberry (Continued)

\begin{tabular}{|c|c|c|c|c|c|c|c|}
\hline \multirow[t]{2}{*}{23} & MESTSSR56F & CATTGCGTTCCTTGAG & ES448442.1 & 220 & ATCATG & 58.8 & Perfect \\
\hline & MESTSSR56R & GGAGCCAAGACTCCTAAG & & & & & \\
\hline \multirow[t]{2}{*}{24} & MESTSSR59F & GAGCTCCGACGACCAC & ES448462.1 & 236 & TCATGA & 54.8 & Perfect \\
\hline & MESTSSR59R & GCGTCTCGACGTGAGAAATAAC & & & & & \\
\hline \multirow{2}{*}{25} & MESTSSR61F & CCATAGCCTCAACGTTTC & ES448534.1 & 239 & AAAAAC & 54.8 & Perfect \\
\hline & MESTSSR61R & CGCTCACGTCCGTATC & & & & & \\
\hline \multirow[t]{2}{*}{26} & MESTSSR66F & GGAAAATTCATCCCCCAAGC & ES448761.1 & 258 & TाTाG & 53.8 & Perfect \\
\hline & MESTSSR66R & CGATGAGAAGCTCAAGGAG & & & & & \\
\hline \multirow[t]{2}{*}{27} & MESTSSR67F & GTGCTCGTAGCTTTGATGG & ES448763.1 & 215 & ATCGCC & 54.8 & Perfect \\
\hline & MESTSSR67R & GCGAAGGAGAAGGAGGAGAG & & & & & \\
\hline \multirow[t]{2}{*}{28} & MESTSSR73F & CTCAAGCTATGCATCCAACGC & ES448909.1 & 237 & CT & 52.8 & Perfect \\
\hline & MESTSSR73R & CCACTTCGAGAGCTTCG & & & & & \\
\hline \multirow[t]{2}{*}{29} & MESTSSR74F & CCATGGCTGAGCACGAG & ES448909.1 & 238 & GAA, GAG & 52.8 & compound \\
\hline & MESTSSR74R & GAGCTCCAGTGTTCCTC & & & & & \\
\hline \multirow[t]{2}{*}{30} & MESTSSR76F & GATCCAGAACTCCCAAACC & ES448912.1 & 209 & CTCCGT & 50.4 & Perfect \\
\hline & MESTSSR76R & GGTAATCCGAGTTCGAGACG & & & & & \\
\hline \multirow[t]{2}{*}{31} & MESTSSR77F & CCATAGCCTCAACGTTTC & ES448915.1 & 238 & AAAAAC & 52.8 & Perfect \\
\hline & MESTSSR77R & CGCTCACGTCCGTATC & & & & & \\
\hline \multirow[t]{2}{*}{32} & MESTSSR78F & GCACTCTCAAACAAATCCTC & ES448921.1 & 242 & AAGTGG & 52.8 & Perfect \\
\hline & MESTSSR78R & CGTTTGGAAACGGCTACTTC & & & & & \\
\hline \multirow[t]{2}{*}{33} & MESTSSR79F & CCCATAGCCTCAACGTTTC & ES448926.1 & 221 & AAAAAC & 45.9 & Perfect \\
\hline & MESTSSR79R & CGACAACAACCGTCAAGTC & & & & & \\
\hline \multirow[t]{2}{*}{34} & MESTSSR85F & GTCATCTATGTCGGGTGGTC & ES448670.1 & 310 & ATACAT & 55.4 & Perfect \\
\hline & MESTSSR85R & CATGGAGCGTTTGTTGTGTG & & & & & \\
\hline \multirow[t]{2}{*}{35} & MESTSSR99F & GGCCAACATGTACGGATAG & ES448967.1 & 203 & CCTAAC & 50.4 & Perfect \\
\hline & MESTSSR99R & CGCCAGGTACAACAAGAAG & & & & & \\
\hline \multirow[t]{2}{*}{36} & MESTSSR108F & GGCTCTGAATGTCCGAGAAG & ES448289.1 & 246 & GAGTTG & 50.4 & Perfect \\
\hline & MESTSSR108R & GGGTGGTAGATTTGGCAC & & & & & \\
\hline \multirow[t]{2}{*}{37} & MESTSSR109F & CTCACGTCCGTATCATCG & ES448314.1 & 244 & TTGTT & 50.4 & Perfect \\
\hline & MESTSSR109R & CCATTCCCATAGCCTCAAC & & & & & \\
\hline \multirow[t]{2}{*}{38} & MESTSSR111F & CATCTATGTCGGGTGGTCG & ES449122.1 & 299 & AAAT & 45.9 & Perfect \\
\hline & MESTSSR111R & CTATGCACAACAGGCTGC & & & & & \\
\hline \multirow[t]{2}{*}{39} & MESTSSR113F & GCCTCCCATTATGCACTATG & ES449132.1 & 206 & AAAACA & 52.8 & Perfect \\
\hline & MESTSSR113R & CGGATCTTCCAGGCTC & & & & & \\
\hline \multirow[t]{2}{*}{40} & MESTSSR115F & CAGGAATCAGAGCCAGAGC & ES448647.1 & 398 & AAAAAC & 53.8 & Perfect \\
\hline & MESTSSR115R & CTGGACCATGTGGAAGC & & & & & \\
\hline \multirow[t]{2}{*}{41} & MESTSSR117F & CATTATCCGGAACACCAGACG & ES448396.1 & 247 & CGG & 53.8 & Perfect \\
\hline & MESTSSR117R & GCTAAGAACCTCGCTCG & & & & & \\
\hline \multirow[t]{2}{*}{42} & MESTSSR121F & CACGTCCGTATCATCGG & ES449197.1 & 244 & TTGTT & 52.8 & Perfect \\
\hline & MESTSSR121R & CCATTCCCATAGCCTCAAC & & & & & \\
\hline \multirow[t]{2}{*}{43} & MESTSSR129F & GATTACTCCAACCAACTCC & ES449040.1 & 223 & AAAACC & 52.8 & Perfect \\
\hline & MESTSSR129R & CAAGGGGGCTAGGAAG & & & & & \\
\hline \multirow[t]{2}{*}{44} & MESTSSR123F & CATCTATGTCGGGTGGTCG & ES448449.1 & 240 & CT & 52.8 & Perfect \\
\hline & MESTSSR123R & GTGTTTGCTGGACTTTGC & & & & & \\
\hline
\end{tabular}


Table 3 Details of the genic (EST) SSR markers developed for mulberry (Continued)

\begin{tabular}{|c|c|c|c|c|c|c|c|}
\hline \multirow[t]{2}{*}{45} & MESTSSR126F & CACCGATGAGCCCTGGTC & ES448693.1 & 200 & $\pi \mathrm{TC}$ & 52.8 & Perfect \\
\hline & MESTSSR126R & GCACAATCCATCCCAAGTG & & & & & \\
\hline \multirow[t]{2}{*}{46} & MESTSSR127F & CCAACATTATCCGGAACACC & ES448594.1 & 285 & CGG & 52.8 & Perfect \\
\hline & MESTSSR127R & CCTGGACGGAAGAAGTGG & & & & & \\
\hline \multirow[t]{2}{*}{47} & MESTSSR131F & CCTCATTGCGTTCCTTGAG & ES448442.1 & 225 & ATA, ATCATG & 54.1 & compound \\
\hline & MESTSSR131R & CTGATTTGGGAGCCAAGAC & & & & & \\
\hline \multirow[t]{2}{*}{48} & MESTSSR132F & CTATGTCGGGTGGTCG & GT735086.1 & 473 & TाTCC & 54.1 & Perfect \\
\hline & MESTSSR132R & CATACCGTCGGAGATGC & & & & & \\
\hline \multirow[t]{2}{*}{49} & MESTSSR136F & CCATTCCCATAGCCTC & ES449178.1 & 244 & AAAAAC & 50.5 & Perfect \\
\hline & MESTSSR136R & CGTCCGTATCATCGG & & & & & \\
\hline \multirow[t]{2}{*}{50} & MESTSSR134F & GGTTGTTGTCGAATCCG & ES448600.1 & 208 & TTGTT & 55.4 & Perfect \\
\hline & MESTSSR134R & GTACAAACCGAACGGGAAC & & & & & \\
\hline \multirow[t]{2}{*}{51} & MESTSSR135F & CCTCATTGCGTTCCTTG & ES448442.1 & 219 & ATCATG & 54.1 & Perfect \\
\hline & MESTSSR135R & CCGGTGAGGTGATTGG & & & & & \\
\hline
\end{tabular}

of 936 alleles were obtained from 188 markers of which 164 (87\%) were polymorphic among the mulberry species and genotypes. These markers revealed an allelic diversity ranging from 1 to 17 with an average of 4.97 alleles per marker locus (Figure 2/Table 6). Earlier reports on allelic diversity of mulberry SSR markers had revealed an average of 4.9 [18], 5.1 [53] and 18.6 [17] alleles per locus. This allelic diversity can be effectively used for various applications ranging from diversity, evolutionary history and QTL mapping of complex traits in mulberry.

Table 4 Various mulberry species (A), mulberry genotypes (B) and other related species (C) for characterizing SSR markers

\begin{tabular}{|c|c|c|c|c|c|}
\hline S.No & Genotypes & Family & Origin & Ploidy & \\
\hline 1 & M. alba & Moraceae & Japan & $2 n=28$ & $\bar{A}$ \\
\hline 2 & M. assambola & Moraceae & - & - & \\
\hline 3 & M. exotica & Moraceae & Zimbabwe & - & \\
\hline 4 & M. indica & Moraceae & India & $2 n=28$ & \\
\hline 5 & M. lavigata & Moraceae & India & $2 n=3 x=42$ & \\
\hline 6 & M. macroura & Moraceae & - & - & \\
\hline 7 & M. multicaulis & Moraceae & China & $2 n=28$ & \\
\hline 8 & Dudia white & Moraceae & India & - & B \\
\hline 9 & Himachal Local & Moraceae & India & - & \\
\hline 10 & MS3 & Moraceae & India & - & \\
\hline 11 & UP105 & Moraceae & India & - & \\
\hline 12 & $\begin{array}{l}\text { Artocarpus heterophyllus } \\
\text { (Jackfruit) }\end{array}$ & Moraceae & Asia & $2 n=56$ & C \\
\hline 13 & Ficus bengalensis (Banyan) & Moraceae & South Asia & - & \\
\hline 14 & Ficus carica (Fig) & Moraceae & South Asia & $2 n=26$ & \\
\hline
\end{tabular}

(Note: All species belong to family Moraceae).
While most of the markers developed in the study amplified the genomic DNA of all mulberry species and genotypes, a few also included private or rare alleles. For instance, Mul3SSR153 only could amplify a few particular mulberry species ( $M$. lavigata, $M$. assambola) and a mulberry genotype (Dudia white). Such private/rare alleles have great utility in establishing the genetic authenticity of a particular species and/or genotype in germplasm characterization as well as in genetic screening experiments [54].

Most of the genic and genomic SSR markers developed in this study were highly informative with an average PIC value of 0.543 which ranged from 0.000 to 0.929 among mulberry species and genotypes (Table 6). Percentage of variation explained by the principal component analysis also revealed that $41 \%$ of the markers were effective in discriminating the variation among the mulberry species and genotypes confirming their efficiency in detecting genetic variations even among closely related varieties.

Two mulberry genotypes viz., Dudia white and UP105 were identified as contrasting lines differing in root traits and WUE in earlier studies [50]. These lines were crossed and a $F_{1}$ segregating population was developed. Of the 188 markers examined, 94 genomic and 22 genic markers were found to be polymorphic between these two parents. These polymorphic markers would be a very useful genomic resource for constructing a genetic linkage map for mulberry. This work is in progress and when done would lead to the determination of the linkage between markers and their position on mulberry linkage groups.

In the present investigation, we report a large number of genic and genomic SSR markers that can be exploited to examine the diversity among mulberry genotypes and 
Table 5 Markers developed for mulberry and their transferability to related species

\begin{tabular}{|c|c|c|c|c|c|c|c|c|}
\hline \multirow[t]{2}{*}{ SSR type } & \multirow{2}{*}{$\begin{array}{l}\text { Locus } \\
\text { specific }\end{array}$} & \multirow{2}{*}{$\begin{array}{l}\text { Monomorpic } \\
\text { in Morus spp }\end{array}$} & \multirow{2}{*}{$\begin{array}{l}\text { Monomorpic } \\
\text { in all species }\end{array}$} & \multirow{2}{*}{$\begin{array}{l}\text { Polymorphic } \\
\text { in Morus spp }\end{array}$} & \multirow{2}{*}{$\begin{array}{l}\text { Primers transferable } \\
\text { to other species }\end{array}$} & \multicolumn{3}{|c|}{ Transferability } \\
\hline & & & & & & Jackfruit & Ficus & Fig \\
\hline Genomic & 137 & 12 & 1 & $125(91.24 \%)$ & 107 (78.10\%) & 96 (70.07\%) & $64(46.71 \%)$ & $64(46.71 \%)$ \\
\hline Genic & 51 & 12 & 6 & 39 (76.47\%) & 42 (82.35\%) & 39 (76.47\%) & 21 (41.17\%) & $22(43.13 \%)$ \\
\hline Total & 188 & 24 & 7 & 164 (87.23\%) & $149(79.25 \%)$ & 135 (71.80\%) & $85(45.21 \%)$ & $86(45.74 \%)$ \\
\hline
\end{tabular}

species. However, the relevance of the marker system would increase if they are transferable to other species.

Transferability of the SSR markers to other related species The transferability of the mulberry SSR markers was examined using three species belonging to the family Moraceae viz., Ficus (F.bengalensis), Fig (F. carica), and Jackfruit (A. heterophyllus) (Table 4). Of all the markers evaluated $78 \%$ (107) genomic and $82 \%$ genic (42) markers showed locus specific amplification in at least one of the three species studied (Table 5). Around $30 \%$ of the markers were transferable to all the three species. Of the 107 genomic and 42 genic markers, 70\% and 76\% were transferable to jackfruit. The transferability of these markers was relatively low in Fig and Ficus, which ranged between 41 to $46 \%$ (Table 5 ). It can be perceived that the genic regions of related genomes would be more conserved than the non-coding regions and hence would have higher transferability [55]. These markers would be highly useful for genome mapping and comparative genomics in mulberry and other closely related species belonging to Moraceae.

Several reports confirm the molecular relatedness of mulberry with a few other plant species belonging to

\begin{tabular}{|c|c|c|c|c|c|}
\hline Samples & Range & $\begin{array}{l}\text { Genetic } \\
\text { diversity }\end{array}$ & $\begin{array}{l}\text { No. of } \\
\text { alleles }\end{array}$ & Heterozygosity & PIC \\
\hline \multirow{3}{*}{$\begin{array}{l}\text { All species and } \\
\text { genotypes }\end{array}$} & Min & 0.0799 & 2 & 0.000 & 0.0767 \\
\hline & Max & 0.9464 & 22 & 0.9091 & 0.9438 \\
\hline & Mean & 0.5969 & 5.47 & 0.1830 & 0.5592 \\
\hline \multirow[t]{3}{*}{ Morus species only } & Min & 0.0000 & 1 & 0.000 & 0.0000 \\
\hline & Max & 0.9339 & 17 & 1.0000 & 0.9299 \\
\hline & Mean & 0.5860 & 4.97 & 0.1881 & 0.5431 \\
\hline \multirow{3}{*}{$\begin{array}{l}\text { Other related } \\
\text { species }\end{array}$} & Min & 0.0000 & 2 & 0.0000 & 0.0000 \\
\hline & Max & 0.8333 & 6 & 1.0000 & 0.8102 \\
\hline & Mean & 0.4090 & 2.57 & 0.0532 & 0.3457 \\
\hline
\end{tabular}

the family Moraceae [56,57]. Thus, the effective transferability of both genic and genomic SSR markers to these species can be expected. In this context, the present study is significant as a large proportion of the mulberry markers were found to be effectively transferable to these closely related species of family Moraceae.

\section{Diversity analysis}

Genetic diversity among the mulberry and three closely related species from the family Moraceae was analyzed using the 188 locus specific markers. We used two clustering algorithms viz., Unweighted Neighbor Joining (NJ) and factorial analysis (FA) to group the species and genotypes. The results of genetic relationships among the species and mulberry genotypes based on NJ and FA is presented in Figures 3 and 4. Both the algorithms were congruent and grouped the species and genotypes into four clusters. A. heterophyllus, F. bengalensis and $F$. carica segregated into a distinct cluster (I) while other mulberry species and genotypes clustered separately (II, III and IV). It was interesting to note that Dudia white clustered along with $M$. lavigata and M. assambola, while all other mulberry species and genotypes grouped into clusters III and IV. Though the dendrogram in Figure 3 indicates clusters III and IV as different, based on the boot strap values, these clusters could be considered as not significantly distinct. Therefore it is apparent that all the mulberry genotypes and species share common alleles except the genotype Dudia white and mulberry species $M$. lavigata and $M$. assambola. The diversity structure represented by the factorial analysis also indicated a similar grouping pattern for the mulberry species and genotypes (Figure 4). Though Dudia white is often considered as a genotype of $M$. alba, there is no firm molecular evidence for its origin.

The genetic relatedness of the 14 species and genotypes is explained in the Table 7. Based on the dissimilarity matrix Fig and UP105 showed maximum dissimilarity (93.8\%) and Fig and Ficus showed the least (38\%). Among the mulberry species and genotypes, the minimum genetic dissimilarity (44.4\%) was observed between $M$. alba and $M$. exotica and highest dissimilarity of $74.7 \%$ was found between Dudia white and UP105. 


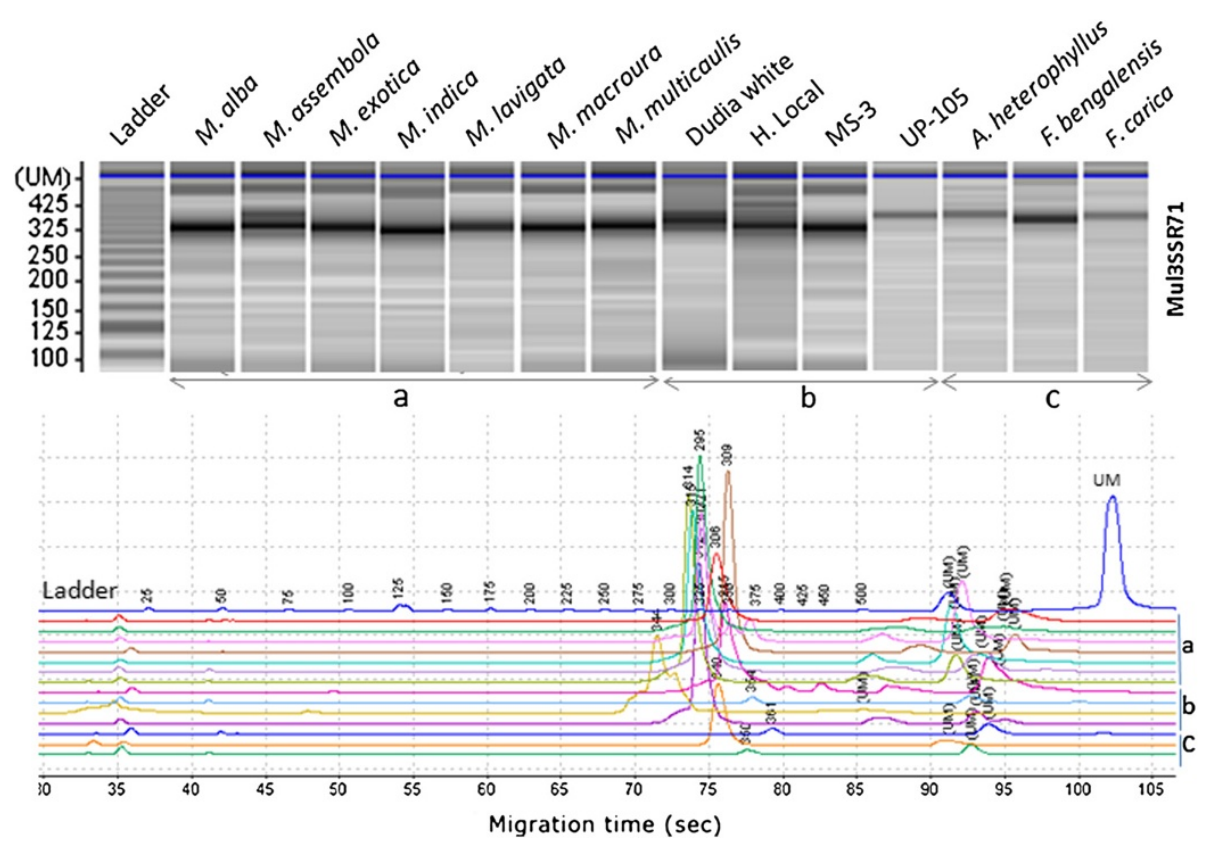

Figure 2 Gel image generated by the MultiNA for different Mulberry species, genotypes and other related species. All species and genotypes belong to family Moraceae. (a) Morus species, (b) Mulberry genotypes and (c) other related species.

These two genotypes significantly differed in physiological traits such as root length and water use efficiency [50].

Overall, the diversity analysis clearly indicates that the markers reported in this study are very well conserved across the taxa and can be effectively utilized to study the genetic relationship among varieties, genotypes and species of Moraceae.

\section{Conclusion}

Considering the commercial importance of mulberry and the complexity of trait based breeding, a focused molecular breeding strategy needs to be evolved for the genetic enhancement of this crop. Lack of sufficient genomic resources such as SSR markers has been one of the major constraints. We report a total of 188 robust locus

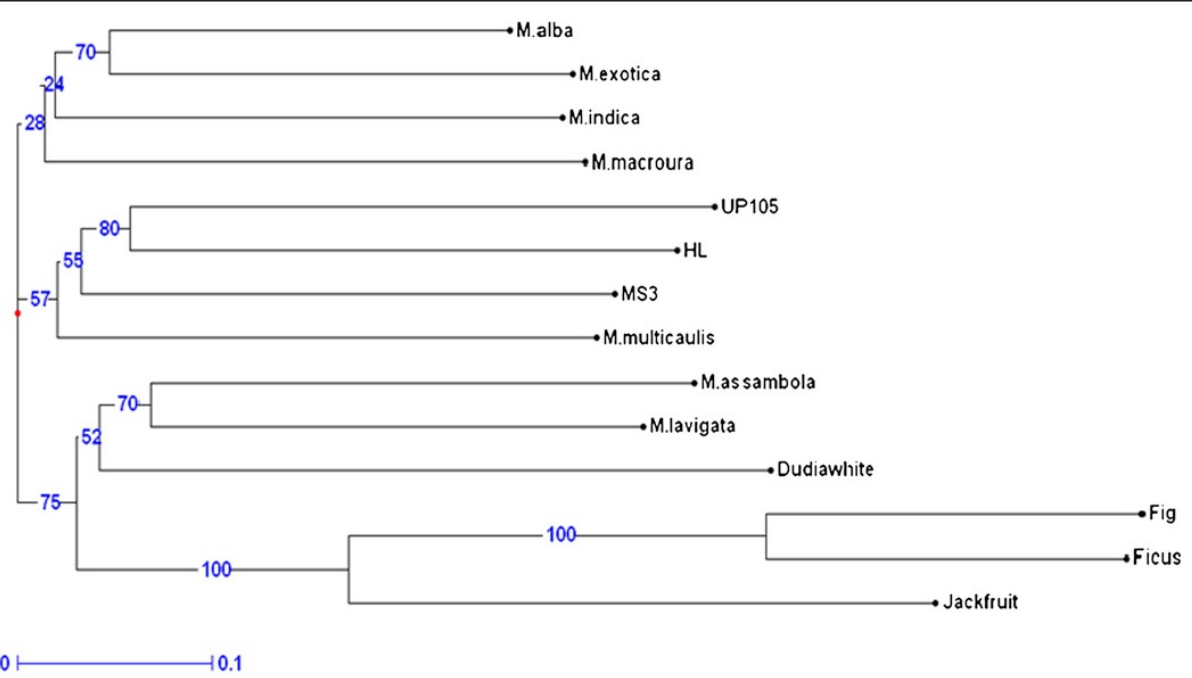

Figure 3 Genetic diversity analysis of mulberry species, genotypes and three related species using both genomic and genic microsatellite markers. Ficus (Ficus bengalensis), Fig (Ficus carica) and Jackfruit (Artocarpus heterophyllus) were the closely related species examined for the transferability of microsatellite markers developed. All species and genotypes belong to family Moraceae. 


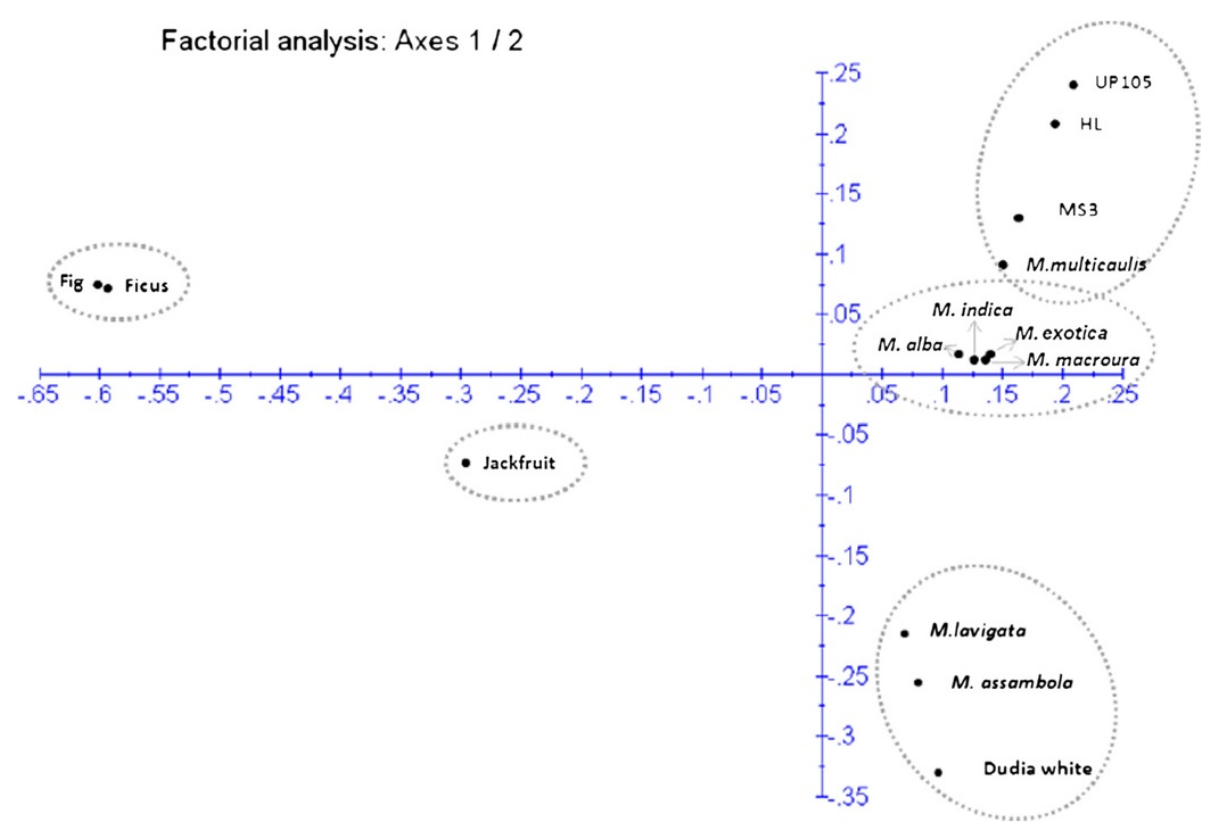

Figure 4 Factorial analysis for grouping of mulberry species, genotypes and three related species using genomic and genic SSR markers. Ficus (Ficus bengalensis), Fig (Ficus carica) and Jackfruit (Artocarpus heterophyllus) were the closely related species examined for the transferability of microsatellite markers developed. All species and genotypes belong to family Moraceae.

specific SSR markers generated by analyzing 3485 genic and genomic sequences of mulberry genome. The markers developed were highly efficient in characterizing seven different mulberry species and four contrasting genotypes of Morus alba L. These markers also exhibited extensive transferability to other related species belonging to the family Moraceae viz., Ficus (Ficus bengalensis), Fig (Ficus carica) and Jackfruit (Artocarpus heterophyllus). The markers displayed high levels of polymorphic information content (PIC) and heterozygosity, enhancing the opportunities of using these markers in diversity analysis as well as for tagging QTLs governing complex agronomic and physiological traits. All the markers developed have been deposited in NCBI/EMBL database and are publicly available.

\section{Methods}

\section{Plant materials and DNA extraction}

Two strategies were adopted for the generation of genomic resources of microsatellite markers for mulberry. Microsatellite motifs in the genomic regions were identified by adopting the pre-cloning enrichment strategy using the genomic DNA isolated from a mulberry genotype Dudia white. Similarly, a stress expressed sequence tag (EST) was analyzed to identify microsatellite motifs in genic regions of mulberry genome. Details of the methodology adopted are described below.
Pre-cloning enrichment strategy for the construction of genomic library and mining of microsatellite motifs

The SSR enriched genomic library was constructed by a modified method of Saghaimaroof et al. [58]. Four micrograms of high quality genomic DNA was extracted from a genotype, Dudia white. This genotype was identified based on the extensive phenotyping carried out with a diverse set of mulberry germplasm [50]. The genomic DNA was digested by blunt-end generating restriction endonuclease, RsaI (MBI Fermentas, USA). This restriction reaction generated a large number of approximately 500-1000 base pair fragments. The ligation of Super SNX linkers, consisting of a Super SNX 24-mer (5'-GTTT AAGGCCTAGCTAGCAGAATC-3') and a phosphorylated 28-mer (5'- pGATTCTGCTAGCTAGGCCTTAAACA AAA-3') to the blunt termini of restriction fragments was performed for 2 hours at $37^{\circ} \mathrm{C}$. To ensure linker ligation, $10 \mu \mathrm{l}$ of digested and ligated product was pre-amplified using $1.5 \mu \mathrm{l}$ of Super SNX24 Forward primer $(10 \mu \mathrm{M}), 150 \mu \mathrm{M}$ of dNTPs, $2 \mathrm{mM} \mathrm{MgCl} 2,1$ unit of Taq DNA polymerase and $25 \mu \mathrm{g} / \mathrm{ml}$ of BSA in a volume of $25 \mu$ l. Self-ligation of the linkers was avoided by adding 1 unit of the restriction enzyme, XmnI. PCR amplification was carried out with a program consisting ofan initial DNA denaturation step of $95^{\circ} \mathrm{C}$ for 2 min followed by 20 cycles of: DNA denaturation step at $95^{\circ} \mathrm{C}$ for $20 \mathrm{~s}$, primer annealing cycle with the appropriate temperature for specific primer pairs for $20 \mathrm{~s}$ and a DNA 
Table 7 Dissimilarity matrix of mulberry and other related species tested for transferability of genic and genomic SSR markers

\begin{tabular}{|c|c|c|c|c|c|c|c|c|c|c|c|c|c|c|}
\hline \multirow[t]{2}{*}{ Accessions } & \multicolumn{7}{|c|}{ Mulberry species } & \multicolumn{4}{|c|}{ Mulberry genotypes } & \multicolumn{3}{|c|}{ Related species } \\
\hline & M. Lavigata & M. indica & M. assambola & M. macroura & M. multicaulis & M. exotica & M. alba & Himachal Local & UP105 & Dudia white & MS3 & Jackfruit & Ficus & Fig \\
\hline M. lavigata & 1 & & & & & & & & & & & & & \\
\hline M.indica & 0.602 & 1 & & & & & & & & & & & & \\
\hline M.assambola & 0.535 & 0.629 & 1 & & & & & & & & & & & \\
\hline M.macroura & 0.615 & 0.544 & 0.641 & 1 & & & & & & & & & & \\
\hline M.multicaulis & 0.620 & 0.578 & 0.647 & 0.590 & 1 & & & & & & & & & \\
\hline M.exotica & 0.608 & 0.527 & 0.634 & 0.550 & 0.584 & 1 & & & & & & & & \\
\hline M.alba & 0.576 & 0.495 & 0.602 & 0.518 & 0.552 & 0.444 & 1 & & & & & & & \\
\hline Himachal local & 0.662 & 0.620 & 0.689 & 0.632 & 0.597 & 0.626 & 0.594 & 1 & & & & & & \\
\hline UP105 & 0.682 & 0.640 & 0.708 & 0.652 & 0.616 & 0.645 & 0.613 & 0.582 & 1 & & & & & \\
\hline Dudia white & 0.625 & 0.668 & 0.651 & 0.680 & 0.686 & 0.673 & 0.641 & 0.728 & 0.747 & 1 & & & & \\
\hline MS3 & 0.630 & 0.587 & 0.656 & 0.600 & 0.564 & 0.593 & 0.561 & 0.581 & 0.600 & 0.695 & 1 & & & \\
\hline Jackfruit & 0.734 & 0.753 & 0.760 & 0.765 & 0.771 & 0.758 & 0.727 & 0.813 & 0.832 & 0.799 & 0.780 & 1 & & \\
\hline Ficus & 0.833 & 0.852 & 0.859 & 0.864 & 0.870 & 0.857 & 0.825 & 0.912 & 0.931 & 0.898 & 0.879 & 0.704 & 1 & \\
\hline Fig & 0.840 & 0.859 & 0.867 & 0.871 & 0.877 & 0.865 & 0.833 & 0.919 & 0.938 & 0.905 & 0.886 & 0.711 & 0.380 & 1 \\
\hline
\end{tabular}


extension cycle of $72^{\circ} \mathrm{C}$ for 2 mins. A final elongation step of $72^{\circ} \mathrm{C}$ for $10 \mathrm{~min}$ was performed to ensure complete amplification of the fragments. All PCR amplifications were carried out using an Eppendorf Master Cycler (Eppendorf, Hamburg). An aliquot of the amplicons was resolved on a $1.2 \%$ agarose gel to check the success of linker ligation.

The restriction digested and linker-ligated DNA fragments were captured by hybridizing with biotinylated microsatellite oligonucleotides (Sigma Aldrich): $[\mathrm{CA}]_{17}$, $[\mathrm{AG}]_{16},[\mathrm{AGC}]_{8},[\mathrm{AGG}]_{8},[\mathrm{ACGC}]_{5},[\mathrm{ACCT}]_{8},[\mathrm{AAC}]_{14}$, $[\mathrm{ATC}]_{14}$ and $[\mathrm{AAG}]_{14}$. The enrichment of microsatellites was carried out in $50 \mu \mathrm{L}$ reaction volume containing $25 \mu \mathrm{L} 2 \times$ hybridization solution $(12 \times$ Sodium saline citrate, $0.2 \%$ SDS), $10 \mu \mathrm{L}$ equimolar biotinylated microsatellite oligos and $2 \mu \mathrm{g}$ of linker ligated DNA. The hybridization of the microsatellite harboring genomic DNA fragments with the biotinylated microsatellite probes was facilitated by a touchdown temperature PCR consisting of 99 cycles of $95^{\circ}$ $\mathrm{C} / 5 \mathrm{~min}, 70^{\circ} \mathrm{C} / 5 \mathrm{sec}, 68.8^{\circ} \mathrm{C} / 5 \mathrm{sec}, 68.6^{\circ} \mathrm{C} / 5 \mathrm{sec}$ with step down of $0.2^{\circ} \mathrm{C}$ for every $5 \mathrm{sec}$ until it reaches $50^{\circ} \mathrm{C}$. The temperature in the tubes was then maintained at $50^{\circ} \mathrm{C}$ for $10 \mathrm{~min}$. Subsequently, a program consisting of 20 cycles of $49.5^{\circ} \mathrm{C} / 5 \mathrm{sec}$ with step down of $0.5^{\circ} \mathrm{C}$ every 5 sec until it reaches $40^{\circ} \mathrm{C} / 5 \mathrm{sec}$ and finally held at $15^{\circ} \mathrm{C}$.

The touchdown PCR conditions facilitate the microsatellite probes to hybridize with complimentary DNA repeat fragments (i.e., expectantly long prefect repeats) when the reaction mixture is at or near the microsatellite probes melting temperature. Hybridized fragments were selectively isolated using Streptavidin coated paramagnetic beads (Roche, Mannheim, Germany). Enriched DNA fragments were amplified with super SNX24 primers and purified using PCR purification column (Sigma, USA). The purified enriched products were ligated to $\mathrm{pTZ} 57 \mathrm{R} / \mathrm{T}$ vector (MBI Fermentas, USA) using T4-DNA ligase overnight at $16^{\circ} \mathrm{C}$. The ligated genomic inserts were cloned in competent $E$. coli DH5 $\alpha$ host cells and grown over night at $37^{\circ} \mathrm{C}$. The transformed colonies were confirmed by performing PCR using M13 universal primers $(3 \mu \mathrm{M})$, $100 \mu \mathrm{M}$ dNTPs, $2 \mathrm{mM} \mathrm{MgCl}$, $1 \mathrm{U}$ Taq DNA polymerase and $1 \mathrm{X}$ PCR buffer, at an annealing temperature of $58^{\circ} \mathrm{C}$ for 30 cycles. PCR products of the recombinant clones were purified using PCR-purification column (Sigma, USA) and sequenced using M13 forward and reverse primers on ABI 3700 sequencer.

\section{Development of EST library to identify genic microsatellite markers}

A stress transcriptome was developed by extracting the total mRNA from the leaves of water stressed and well watered mulberry plants. A widely adopted mulberry variety, K2 was used for this purpose. A modified guanidiumisothiocyanate protocol [59] was adopted to isolate total RNA from mature leaf tissue. Total messenger RNA (mRNA) was then isolated from $1 \mathrm{mg}$ of total RNA using mRNA isolation kit (Promega). The mRNA was reverse transcribed to develop cDNA and the ESTs have been isolated [19]. These EST sequences were used in this investigation to develop genic SSR markers.

\section{SSR marker development}

Initially, the sequences were analyzed to identify unique and non-redundant libraries of genic and genomic regions for designing primers. The nucleotide sequences were analyzed using the Clustal-W, an on-line toolto determine the complemetarity between pairs of sequences. The non-redundant sequences were analyzed with "Mreps" software (http://bioinfo.lifl.fr/mreps/mreps.php) to identify sequences containing microsatellite motifs. The analysis revealed the presence of a single nucleotide base being the repeat motif (mono nucleotide repeat - MNR) to as high as regions with more than six bases (long nucleotide repeat - LNR). The MNR and LNR sequences were omitted from further analysis and primers were designed only the sequences with repeat motifs of two nucleotides (di-nucleotide repeats - DNR) and six nucleotides (hexa-nucleotide repeats - HNR). Primer3, also online software was used for designing appropriate primers [60]. The quality of primers was determined using the FAST PCR program and only those primers that would amplify a fragment in the range of 150 and 450 base pairs of template DNA were selected. Synthesis of these primers was outsourced to Bioserve India Pvt. Ltd., Hyderabad). Each of the primer pairs was standardized for their locus specific amplification using the genomic DNA of Dudia white as a template. GradientPCR was carried out in a total volume of $15 \mu \mathrm{L}$ containing $2 \mathrm{ng}$ of DNA template, $1 \times$ Taq buffer, $2 \mathrm{mM} \mathrm{MgCl}_{2}$, $0.2 \mathrm{mM}$ dNTPs, $1 \mathrm{U}$ Taq DNA polymerase (MBI Fermentas, USA) and $3 \mu \mathrm{M}$ each of forward and reverse primers. Amplification was performed in a epGradient Master cycler (Eppendorf, Hamburg)with the following PCR conditions: DNA denaturation at $95^{\circ} \mathrm{C}$ for $5 \mathrm{~min}$ followed by 30 cycles of $95^{\circ} \mathrm{C}$ for $1 \mathrm{~min}$, primer annealing temperatures ranging between $45-65^{\circ} \mathrm{C}$ for $45 \mathrm{~s}$ (depending on the Ta for each primer pair) and a DNA extension step of $72^{\circ} \mathrm{C}$ for $45 \mathrm{~s}$ and a final extension step at $72^{\circ} \mathrm{C}$ for $8 \mathrm{~min}$. The details of the primer sequences, their annealing temperatures, expected amplicon size etc. are summarized in Table 2 and Table 3. The amplified products were resolved on 3\% agarose gels. Only those primer pairs that produced unambiguous single band amplification alone were considered for the development of SSR markers in mulberry. This stringency ensured the development of robust SSR markers in 
mulberry which can be effectively used for diversity analysis as well as for constructing genetic linkage maps. Only such markers were further used for validation.

\section{Validation of markers}

Each of the markers was examined for their ability in amplifying the genomic DNA from other mulberry species and genotypes. Genomic DNA was extracted from seven distinct mulberry species and four contrasting genotypes of mulberry using a modified CTAB method [61]. These four genotypes were selected based on the extensive phenotyping of a set of 295 germplasm accessions for the variability in root traits and water use efficiency. Thus, the four genotypes represent contrast for these highly relevant drought adaptive traits. The list of the mulberry species and genotypes are given in Table 4 . The template DNA from the different mulberry species and genotypes were amplified using each of the primers for genic and genomic microsatellite markers. The PCR conditions followed are same as that adopted for gradient PCR, explained above. All the amplified products were analyzed on microchip based electrophoresis system MultiNA (Shimadzu biotech, Japan) and the highest peak detected by the fragment analyzer was scored for the presence of the expected band for each primer pair. The polymorphism data was scored and used for the determination of polymorphic information content (PIC) for each marker as per Liu and Muse [62], Observed heterozygosity and allele diversity were computed using the Power Marker 3.25 software [62]. The most appropriate locus specific marker competent to divulge the variation among the species and genotypes was determined by principle component analysis (PCA).

\section{Genetic diversity and cross species transferability}

It is well known that there would be significant levels of sequence homology between closely related species and hence, there would be a possibility of a specific SSR marker detecting a similar locus in other related species. Establishment of the transferability of markers to other related species is therefore important while developing locus specific marker systems. The transferability of these markers was examined in three closely related species belonging to the family Moraceae, namely Ficus (F. bengalensis), Fig (F. carica) and Jackfruit (A. heterophyllus) (Table 4).

The percentage of transferability of the markers was calculated for each species by determining the presence of target loci to the total number of loci analyzed. The allelic diversity data obtained for all the microsatellite loci amplified were used to compute the genetic dissimilarity using DARwin v.5.0 program [63]. The dissimilarity matrix was further used to group the species according to their genetic relatedness based on Unweighted Neighbor Joining method and factorial analysis.

\section{Additional file}

Additional file 1: Marker-wise details of the gene diversity, heterozygosity and PIC values tested using mulberry species and genotypes.

\section{Competing interests}

The authors declare that they have no competing interest.

\section{Authors' contributions}

$\mathrm{MB}$ and NNK contributed equally for the development of microsatellite enriched genomic libraries, marker development and validation. MB wrote the first draft of the manuscript. JB, SHR, MA and MJN contributed in genotyping activity. MSS conceptualized and directed the research program. UKM coordinated the research and overviewed the progress. The EST library was developed and annotated by PK. All authors reviewed and edited the manuscript. All authors read and approved the final manuscript.

\section{Acknowledgement}

This work was carried out with the financial support from Department of Biotechnology (DBT), Government of India to MSS which is sincerely acknowledged (File No: Grant/DBT/CSH/GIA/1395/2010-11. We also wish to thank the Departments of Sericulture and Horticulture, UAS, Bangalore for kindly providing the samples of mulberry and other related species, respectively. We acknowledge the technical inputs and suggestions by Dr. T.K. Narayanaswamy, Professor of Sericulture.

\section{Author details}

${ }^{1}$ Department of Crop Physiology, University of Agricultural Sciences, Bangalore, India. ${ }^{2}$ Department of Sericulture, University of Agricultural Sciences, Bangalore, India. ${ }^{3}$ Department of Plant Molecular Biology, University of Delhi, South Campus, New Delhi, India.

Received: 2 May 2013 Accepted: 13 November 2013

Published: 1 December 2013

\section{References}

1. Kshama G, Mahanta JC, Deole AL, Kantharaju BM: Raw silk production: 2007-08. Indian Silk 2008, 47:43-44

2. Khurana $P$, Checker VG: The advent of genomics in mulberry and perspectives for productivity enhancement. Plant Cell Rep 2011, 30:825-838.

3. Zietjiewicz E, Rafalski A, Labuda D: Genome fingerprinting by simple sequence repeat (SSR) - anchored polymerase chain reaction amplification. Genomics 1994, 20:176-183.

4. Prevost A, Wilkinson MJ: A new system of comparing PCR primers applied to ISSR fingerprinting of potato cultivars. Theor App/ Genet 1999, 98:107-112

5. Gepts P: The use of molecular and biochemical markers in crop evolution studies. Evol. Biol 1993, 27:51-94

6. Mathithumilan B, Dandin SB: Genetic analysis of diploid and colchitetraploid mulberry (Morus indica and Morus alba) by molecular and morphological markers. Int J Plant Breed 2008, 3:58-64.

7. Vijayan K, Chatterjee SN: ISSR profiling of Indian cultivars of mulberry (Morus spp.) and its relevance to breeding programs. Euphytica 2003, 131:53-63.

8. Vijayan K, Srivastava PP, Awasthi AK: Analysis of phylogenetic relationship among five mulberry (Morus) species using molecular markers. Genome 2004, 47:439-448.

9. Awasthi AK, Nagaraja GM, Naik GV, Sriramana K, Thangavelu K, Nagaraju J: Genetic diversity and relationships in mulberry (Genus Morus) as revealed by RAPD and ISSR marker assays. BMC Genet 2004, 5:1-18.

10. Beckmann JS, Soller M: Toward a unified approach to genetic mapping of eukaryotes based on sequence tagged microsatellite sites. Bio Tech 1990, 8:930-932.

11. Tautz D, Renz M: Simple sequences are ubiquitous repetitive components of eukaryotic genomes. Nucl Acid Res 1984, 25:4127-38.

12. Bhat PR, Krishnakumar V, Hendre PS, Rajendrakumar P, Varshney RK, Aggarwal RK: Identification and characterization of expressed sequence tags-derived simple sequence repeats markers from robusta coffee variety 
'CXR' (an interspecific hybrid of Coffea canephora x Coffeacongensis). Mol Ecol Notes 2005, 5:80-83.

13. McCouch SR, Teytelman L, Xu Y, Lobos KB, Clare K, Walton M, Fu B, Maghirang R, Li Z, Xing Y, Zhang Q, Kono I, Yano M, Fjellstrom R, De Clerck G, Schneider D, Cartinhour S, Ware D, Stein L: Development and Mapping of 2240 New SSR Markers for Rice (Oryza sativa L.). DNA Res 2002, 9:199-207.

14. Abbott AG, Sosinski BM, Gannavarapu LD, Hager LE, Beck GJ, King CD, Ryder S, Rajapakse WW, Baird RE, Ballard: Characterization of microsatellite markers in peach (Prunuspersica (L.) Batsch). Theor Appl. Genet 2000, 101:421-428.

15. Mookerjee S, Guerin J, Collins G, Ford C, Sedgley M: Paternity analysis using microsatellite markers to identify pollen donors in an olive grove. Theor Appl Genet 2005, 111:1174-1182.

16. Giraldo E, Viruel MA, Opez-Corrales ML, Hormaza Jl: Characterization and cross-species transferability of microsatellites in the common fig (Ficus carica L.). J Hort Sci Biotechnol 2005, 80:217-224.

17. Aggarwal RK, Udaykumar D, Hendre PS, Sarkar A, Singh LI: Isolation and characterization of six novel microsatellite markers for mulberry (Morus indica). Mol Ecol Notes 2004, 4:477-479.

18. Zhao W, Miao X, Jia S, Pan Y, Huang Y: Isolation and characterization of microsatellite loci from the mulberry, Morus L. Plant Science 2005, 168:519-525.

19. Khurana P, Khurana JP, Ravi V, Lal S: Repertoire of leaf expressed sequence tags (ESTs) and partial characterization of stress-related and membrane transporter genes from mulberry(Morus indica L.). Tree Genet Genomes 2009, 5(2):359-374.

20. Gulyani V, Khurana P: Identification and expression profiling of droughtregulated genes in mulberry (Morus sp.) by suppression subtractive hybridization of susceptible and tolerant cultivars. Tree Genet Genomes 2011, 7:725-738

21. Checker VG, Saeed B, Khurana P: Analysis of expressed sequence tags from mulberry (Morus indica) roots and implications for comparative transcriptomics and marker identification. Tree Genet Genomes 2012, 8:1437-1450.

22. Li Y, Korol AB, Fahima T, Beiles A, Nevo E: Microsatellites within genes: structure, function, and evolution. Mol Biol Evol 2004, 6:991-1007.

23. Lagercrantz $U$, Ellegren $H$, Andersson $L$ : The abundance of various polymorphic SSR motifs differs between plants and vertebrates. Nucl Acid Res 1993, 21:1111-1115.

24. Morgante M, Olivieri AM: PCR-amplified SSRs as markers in plant genetics. Plant J. 1993, 3:175-182

25. Wang Z, Weber JL, Zhong G, Tanksley SD: Survey of plant short tandem DNA repeats. Theor Appl Genet 1994, 88:1-6.

26. Liu BH: Statistical Genomics: In Linkage Mapping and QTL Analysis. Boca Raton: CRC Press, FA; 1997

27. Edwards KJ, Barker JHA, Daly A, Jones C, Karp A: Microsatellite libraries enriched for several microsatellite sequences in plants. Biotechniques 1996, 20:758-760

28. Zane $\mathrm{L}$, Bargelloni $\mathrm{L}$, Patarnello $\mathrm{T}$ : Strategies for microsatellite isolation: a review. Mol Ecol 2002, 11:1-16.

29. Ferguson ME, Burow MD, Schultz SR, Bramel PJ, Paterson AH, Kresovich S, Mitchell S: Microsatellite identification and characterization in peanut (A. hypogaea L.). Theor Appl Genet 2004, 108:1064-1070.

30. He G, Meng R, Newman M, Gao G, Pittman RN, Prakash CS: Microsatellites as DNA markers in cultivated peanut (Arachis hypogaea L.). BMC Plant Biol 2003, 3:3-9.

31. Jung S, Abbott A, Jesudurai C, Tomkins J, Main D: Frequency, type, distribution and annotation of simple sequence repeats in Rosaceae ESTs. Funct Integr Genomics 2005, 5:136-143.

32. Aggarwal RK, Hendre PS, Varshney RK, Bhat PR, Krishna kumar V, Singh L: Identification, characterization and utilization of EST-derived genic microsatellite markers for genome analyses of coffee and related species. Theor Appl Genet 2007, 114:359-372.

33. Feng SP, Li WG, Huang HS, Wang JY, Wu YT: Development, characterization and cross-species/genera transferability of ESTSSR markers for rubber tree (Hevea brasiliensis). Mol Breed 2009, 23:85-97.

34. Nicot N, Chiquet V, Gandon B, Amilhat L, Legeai F, Leroy P, Bernard M, Sourdille P: Study of simple sequence repeat (SSR) markers from wheat expressed sequence tags (ESTs). Theor Appl Genet 2004, 109:800-805.

35. Gao LF, Tang JF, Li HW, Jia JZ: Analysis of microsatellites in major crops assessed by computational and experimental approaches. Mol Breed 2003, 12:245-261.
36. Moretzsohn MC, Leoi L, Proite K, Guimaraes PM, Leal-Bertioli SCM, Gimanes MA, Martin WS, Valls JFM, Grattapaglia D, Bertioli D: A microsatellite based, gene-rich linkage map for the AA genome of Arachis (Fabaceae). Theor Appl Genet 2005, 111:1060-1071.

37. Gimenes MA, Hosino AA, Barbosa AVG, Palmieri DA, Lopes CR: Characterization and transferability of microsatellite markers of cultivated peanut (Arachis hypogaea). BMC Plant Biol 2007, 7:9.

38. Butcher PA, Decrooca S, Gray Y, Moran GF: Development, inheritance and cross-species amplification of microsatellite markers from Acacia mangium. Theor. Appl. Genet 2000, 101:1282-1290.

39. De Wiel CV, Arens P, Vosman B: Microsatellite retrieval in lettuce (Lactuca sativa L.). Genome 1998, 42:139-150.

40. Cuc LM, Mace ES, Crouch JH, Quang VD, Long TD, Varshney R: Isolation and characterization of novel microsatellite markers and their application for diversity assessment in cultivated groundnut (Arachis hypogaea). BMC Plant Biol 2008, 8:55

41. Weber JL: Informativeness of human (dC-dA)n (dG-dT)n polymorphism. Genomics 1990, 7:524-530.

42. Hopkins MS, Casa AM, Wang T, Mitchell SE, Dean RE, Kochertgandkresovich S: Discovery and characterization of polymorphic simple sequence repeats (SSRs) in peanut. Crop Sci 1999, 39:1243-1247.

43. Scotti I, Vendramin GG, Matteotti LS, Scarponi C, Sari-Gorla M, Binelli G: Postglacial recolonization routes for Piceaabies K. in Italy as suggested by the analysis of sequence-characterized amplified region (SCAR) markers. Mol Ecol 2000, 9:699-708.

44. Cordeiro GM, Casu R, McIntyre CL, Manners JM, Henry RJ: Microsatellite markers from sugarcane (Saccharum spp.) ESTs cross transferable to Erianthus and Sorghum. Plant Sci 2001, 160:1115-1123.

45. Metzgar D, Bytof J, Wills C: Selection against frame shift mutations limits microsatellite expansion in coding DNA. Genome Research 2000, 10:72-80.

46. Morgante $M$, Hanafey $M$, Powell W: Microsatellites are preferentially associated with non-repetitive DNA in plant genomes. Nat. Genet 2002, 30:194-200

47. Wang Y, Kang M, Huang M: Microsatellite Loci Transferability in Chestnut. J Amer Soc Hort Sci 2008, 133:692-700.

48. Marcotte EM, Pellegrini M, Yeates TO, Eisenberg D: A census of protein repeats. J Mol Biol 1999, 293:151-160.

49. Wren JD, Forgacs E, Fondon JW, Pertsemlidis A, Cheng SY: Repeat polymorphisms within gene regions: Phenotypic and evolutionary implications. Am J Human Genet 2000, 67:345-356.

50. Vinoda KS: Identification of QTLS conditioning water use efficiency and associated traits in mulberny though marker assisted selection. PhD thesis; 2010.

51. Varshney RK, Graner A, Sorrells ME: Genic microsatellite markers in plants: Features and applications. Tren biotech 2005, 23:48-55.

52. Grattapaglia D, Plomion C, Krist M, Sedroff R: Genomics of growth traits in forest tress. Curr Opin Plant Biol 2009, 12:1-9.

53. Zhao WG, Zhou ZH, Miao XX, Zhang Y, Wang SB, Huang $J H$, Xiang $H$, Pan $Y L$, Huang YP: A comparison of genetic variation among wild and cultivated Morus species (Moraceae: Morus) as revealed by ISSR and SSR markers. Biodiv Conserv 2007, 6:275-290.

54. Kalinowski ST: Counting alleles with rarefaction: private alleles and hierarchical sampling designs. Conser Genet 2004, 5:539-543.

55. Gupta PK, Rustgi S, Sharma S, Singh R, Kumar N, Balyan HS: Transferable EST-SSR markers for the study of polymorphism and genetic diversity in bread wheat. Mol Genet Genomics 2003, 270:315-323.

56. Datwyler SL, Weiblen GD: On the origin of the Fig: Phylogenetic relationships of Moraceae from NDHF sequences. Am J Bot 2004, 5:767-777.

57. Leitch IJ, Chase MW, Bennett MD: Phylogenetic analysis of DNA C-values provides evidence for a small ancestral genome size in flowering plants. Annal Bot 1998, 82:85-94

58. Saghaimaroof MA, Solima KM, Jorgenson RA, Allard RW: Ribosomal DNA spacer-length polymorphisms in barley: Mendelian inheritance, chromosomal location, and population dynamics. Proceed Nat Acad Sci 1984, 81:8014-8018. USA.

59. Chomczynski P, Sacchi N: Single-step method of RNA isolation by acid guanidiniumthiocyanate-phenol-chloroform extraction. Anal Biochem 1987, 162:156-9.

60. Rozen S, Skaletsky H: Primer3 on the WWW for general users and for biologist programmers. Met Mol Biol 2000, 132:365-386. 
61. Ausubel FM, Brent R, Kingston RE, Moore DD, Seidmann JG, Smith JA, Struhl E: (Eds): Current protocols in molecular biology, Volume 3. New York: John Wiley and Sons; 2003.

62. Liu K, Muse SV: Power Marker: an integrated analysis environment for genetic marker analysis. Bioinformatics 2005, 21:2128-2129.

63. Perrier X, Flori A, Bonnot F: Data analysis methods. In Genetic diversity of cultivated tropical plants. Edited by Hamon P, Seguin M, Perrier X,

Glaszmann JC. Enfield: Science Publishers, Montpellier; 2003:43-76.

doi:10.1186/1471-2229-13-194

Cite this article as: Mathithumilan et al:: Development and

characterization of microsatellite markers for Morus spp. and assessment of their transferability to other closely related species. BMC Plant Biology 2013 13:194.

\section{Submit your next manuscript to BioMed Central and take full advantage of:}

- Convenient online submission

- Thorough peer review

- No space constraints or color figure charges

- Immediate publication on acceptance

- Inclusion in PubMed, CAS, Scopus and Google Scholar

- Research which is freely available for redistribution 